\title{
Acoustic scattering by cascades with complex boundary conditions: compliance, porosity and impedance
}

\author{
Peter J. Baddoo ${ }^{1,} \uparrow$ and Lorna J. Ayton ${ }^{1}$ \\ ${ }^{1}$ Department of Applied Mathematics and Theoretical Physics, Centre for Mathematical Sciences, \\ University of Cambridge, UK
}

(Received 31 August 2019; revised 11 May 2020; accepted 20 May 2020)

We present a solution for the scattered field caused by an incident wave interacting with an infinite cascade of blades with complex boundary conditions. This extends previous studies by allowing the blades to be compliant, porous or satisfy a generalised impedance condition. Beginning with the convected wave equation, we employ Fourier transforms to obtain an integral equation amenable to the Wiener-Hopf method. This Wiener-Hopf system is solved using a method that avoids the factorisation of matrix functions. The Fourier transform is inverted to obtain an expression for the acoustic potential function that is valid throughout the entire domain. We observe that the principal effect of complex boundary conditions is to perturb the zeros of the Wiener-Hopf kernel, which correspond to the duct modes in the inter-blade region. We focus efforts on understanding the role of porosity, and present a range of results on sound transmission and generation. The behaviour of the duct modes is discussed in detail in order to explain the physical mechanisms behind the associated noise reductions. In particular, we show that cut-on duct modes do not exist for arbitrary porosity coefficients. Conversely, the acoustic far-field modes are unchanged by modifications to the boundary conditions. We apply our solution to a cascade of perforated plates and see that a fractional open area of $1 \%$ is sufficient to significantly attenuate backscattering. The solution is essentially analytic (the only numerical requirements are matrix inversion and root finding) and is therefore extremely rapid to compute.

Key words: wave scattering, wave-structure interactions

\section{Introduction}

Turbomachinery noise remains a significant contributor to overall aero-engine noise (Peake \& Parry 2012). A considerable source of broadband noise is the so-called 'rotor-stator interaction', where unsteady wakes shed by compressor rotors interact with downstream stators. Much progress has been made in understanding rotor-stator

$\dagger$ Present address: Department of Mathematics, Huxley Building, South Kensington Campus, Imperial College London, London SW7 2AZ, UK. Email address for correspondence: 
interaction noise when the blades are modelled as flat plates (Peake 1992; Glegg 1999; Posson, Roger \& Moreau 2010; Bouley et al. 2017) or with realistic geometries (Evers \& Peake 2002; Baddoo \& Ayton 2020). However, research into blades with complex boundaries - where the blades are not necessarily impermeable and rigid is far less developed, especially from an analytic standpoint. This paper presents the first analytic treatment of scattering by cascades with complex boundary conditions, including compliance, porosity and impedance.

Aspirations for lighter and more efficient engines have driven the design of thinner and lighter blades in turbomachinery (Saiz 2008). As a result, aeroelastic effects such as flutter and resonance must be considered in modern turbomachinery design and testing (Hall, Kielb \& Thomas 2006). The rapid and accurate prediction of the aeroacoustic performance of turbomachinery with consideration of aeroelastic effects is therefore essential in evaluating the appropriateness of potential blade designs. Analytic solutions are excellent candidates for this task (Peake 1992; Glegg 1999; Posson et al. 2010), but are presently limited to impermeable and rigid blades with no consideration of aeroelastic effects. The present study permits the consideration of compliant blades (Crighton \& Leppington 1970), where the blade deforms with a (purely) local response to the pressure gradient across the blade and elastic restoring forces are ignored. This is particularly relevant to marine applications where inertial effects dominate elastic restoring forces and our solution is an important first step towards modelling more general linearised elastic blades (Cavalieri, Wolf \& Jaworski 2016).

An influential trend in aeroacoustic research is to modify aerofoils with noise reducing technologies. A popular choice is a poroelastic extension, which was originally inspired by the silent flight of owls (Graham 1934), and the corresponding experimental support of Geyer, Sarradj \& Fritzsche (2010). A detailed review of research into the silent flight of owls is available in Jaworski \& Peake (2020). Poroelastic extensions have been applied to semi-infinite (Jaworski \& Peake 2013) and finite (Ayton 2016) blades and have demonstrated considerable noise reductions. Structural requirements limit the application of highly porous blades in turbomachinery, but there remains the possibility that significant noise reductions are available for modest porosity values. The approach of the present research permits porous blades through the assumption of an impedance condition where the seepage velocity through the blade is proportional to the pressure jump across the blade. We will also consider the more general situation of an impedance relation along the blades (Myers 1980).

In this paper we extend the analyses of Glegg (1999) and Posson et al. (2010) to analyse the scattering by a cascade of blades with a range of boundary conditions. The problem is solved with tools from complex variable theory, particularly the Wiener-Hopf method. Taking a Fourier transform maps the problem into the spectral plane where the Wiener-Hopf analysis is carried out in a similar way to Glegg (1999). An inverse Fourier transform is applied to return the problem to physical space, and contour integration is applied to recover the acoustic field (Posson et al. 2010). A significant advantage of the presented technique is that the method is identical regardless of the boundary condition - the only effects of modifying the boundary condition are to modify the kernel in the Wiener-Hopf method.

A striking feature of the analysis is that modifications to the boundary conditions do not affect the modal structure of the solution in the far field. We arrive at this result by interrogating the (meromorphic) Wiener-Hopf kernel, which takes an interpretable form amenable to asymptotic and numerical analysis. We show that, in 
the spectral plane, the only effect of modifying the blade boundary condition is to vary the locations of the zeros of this Wiener-Hopf kernel, which corresponds to perturbing the duct modes of the cascade response function. Modifying the zeros has a significant effect on the acoustic field in the inter-blade region, in part because the cut-on frequencies of the duct modes are changed to account for energy being absorbed or produced by the blades. In fact, we will show that, in the case where the blades are porous, the duct modes are never cut-on. The poles of the Wiener-Hopf kernel correspond to the acoustic modes scattered into the far field and are invariant under modifications to the boundary conditions; the modes are a function of the cascade spacing and incident field alone. Consequently, the cut-on frequencies of the acoustic modes are unchanged and the modal structure of the upstream and downstream acoustic fields are the same regardless of boundary condition, although the coefficients of these modes do change, thus permitting a far-field noise reduction.

Our analysis is focussed on unloaded flat plates, although it is well known that loaded blades have a significant effect on aeroacoustic performance (Fang \& Atassi 1993; Evers \& Peake 2002; Hall et al. 2006). The present work could be merged with our recent solution for scattering by loaded cascades (Baddoo \& Ayton 2020) to account for both the effects of complex boundary conditions and non-trivial geometries.

In the present study we consider four possible boundary conditions for the cascade blades, labelled cases 0-III. Physically, case 0 corresponds to rigid and impermeable blades; case I, porous or compliant blades with no background flow; case II, porous blades with background flow; and case III, a general impedance relation. Mathematically, case 0 corresponds to a Neumann boundary condition; case I, a Robin boundary condition; case II, an oblique derivative boundary condition; and case III, a generalised Cauchy boundary condition.

We begin by presenting a mathematical model for the cascade in $\S 2$, including the modelling of the various boundary conditions. We then present some details of the mathematical solution in $\S 3$. In $\S 4$ we conduct a detailed investigation of the effects of blade porosity. In particular, we present a range of results on sound generation and sound transmission. Finally, in $\S 5$ we summarise our work and suggest future directions of research. The code used to produce all the results is available at https://github.com/baddoo/complex-cascade-scattering.

\section{Mathematical formulation}

We consider a rectilinear cascade of blades in a uniform, subsonic flow as illustrated in figure 1. It is useful to rotate the coordinate system and define

$$
\left(x^{*}, y^{*}, z^{*}\right)=\left(\tilde{x} \cos \left(\chi^{*}\right)+\tilde{y} \sin \left(\chi^{*}\right),-\tilde{x} \sin \left(\chi^{*}\right)+\tilde{y} \cos \left(\chi^{*}\right), \tilde{z}\right),
$$

so that the $x^{*}$ and $y^{*}$ coordinates are tangential and normal to the blades respectively, which have dimensional length $2 b^{*}$. The blades are unloaded, but we do permit a background flow tangential to the blades. Additionally, the background flow may have a spanwise component such that $\boldsymbol{U}_{0}^{*}=\left(U_{0}^{*}, 0, W_{0}^{*}\right)$, as illustrated in figure 2 . The blades in the cascade are inclined at stagger angle $\chi^{*}$, and the distance between adjacent blades is $\Delta^{*}$. Consequently, the spacing between blades is given by

$$
\left(d^{*}, s^{*}\right)=\Delta^{*}\left(\sin \left(\chi^{*}\right), \cos \left(\chi^{*}\right)\right) .
$$

We assume, as it is often the case in practical scenarios, that the blades are staggered and overlapping i.e. $0<d^{*}<2 b^{*}$. The case of non-overlapping blades could be treated 

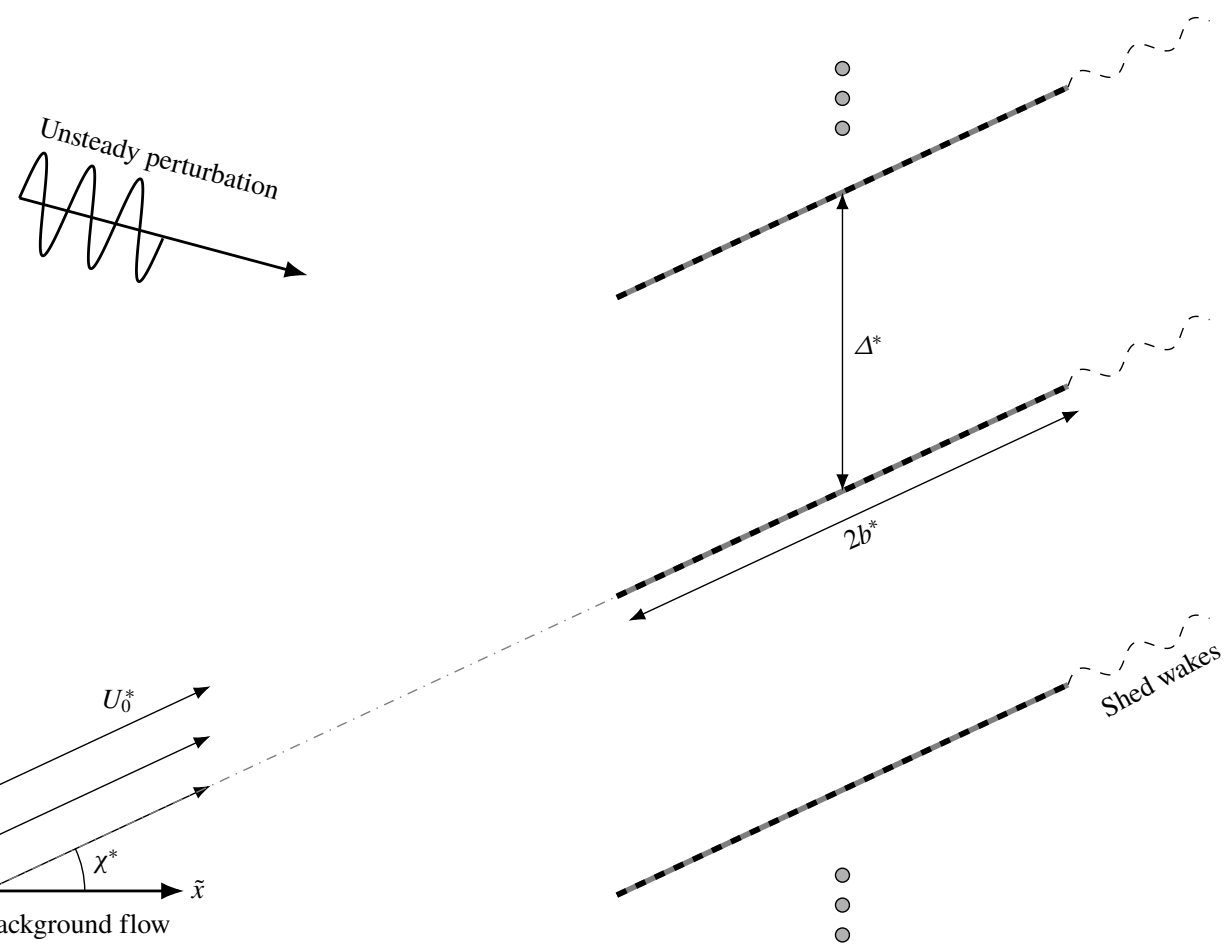

FIGURE 1. An infinite, rectilinear cascade of flat plates with complex boundary conditions subjected to an unsteady perturbation. The plates have dimensional length $2 b^{*}$ and are inclined at a stagger angle of $\chi^{*}$. The plates produce an unsteady wake in the case where there is a non-zero chordwise background flow $U_{0}^{*}$.

in a similar fashion to Maierhofer \& Peake (2020). The zero-stagger arrangement can be recovered as a degenerate case of our results. We further assume that a vortical or acoustic wave is incident on the cascade, resulting in a velocity perturbation $\boldsymbol{u}^{*}$ to the mean flow. The Kutta condition is satisfied by ensuring that there is no pressure jump at the blades' trailing edges, and the pressure singularity at the leading edge is restricted to be integrable.

We introduce an acoustic potential function for the scattered field defined by

$$
\nabla \phi^{*}=u^{*} \text {. }
$$

Consequently, conservation of momentum gives the scattered pressure as

$$
p^{*}=-\rho_{0}^{*} \frac{\mathrm{D}_{0} \phi^{*}}{\mathrm{D}_{0} t^{*}},
$$

where $\rho_{0}^{*}$ is the mean flow density and the (linearised) convective derivative is defined as

$$
\frac{\mathrm{D}_{0}}{\mathrm{D}_{0} t^{*}}=\frac{\partial}{\partial t^{*}}+\boldsymbol{U}_{0}^{*} \cdot \nabla=\frac{\partial}{\partial t^{*}}+U_{0}^{*} \frac{\partial}{\partial x^{*}}+W_{0}^{*} \frac{\partial}{\partial z^{*}} .
$$

Accordingly, conservation of mass gives the convected wave equation

$$
\frac{1}{c_{0}^{2}} \frac{\mathrm{D}_{0}^{2} \phi^{*}}{\mathrm{D}_{0} t^{* 2}}-\nabla^{2} \phi^{*}=0,
$$




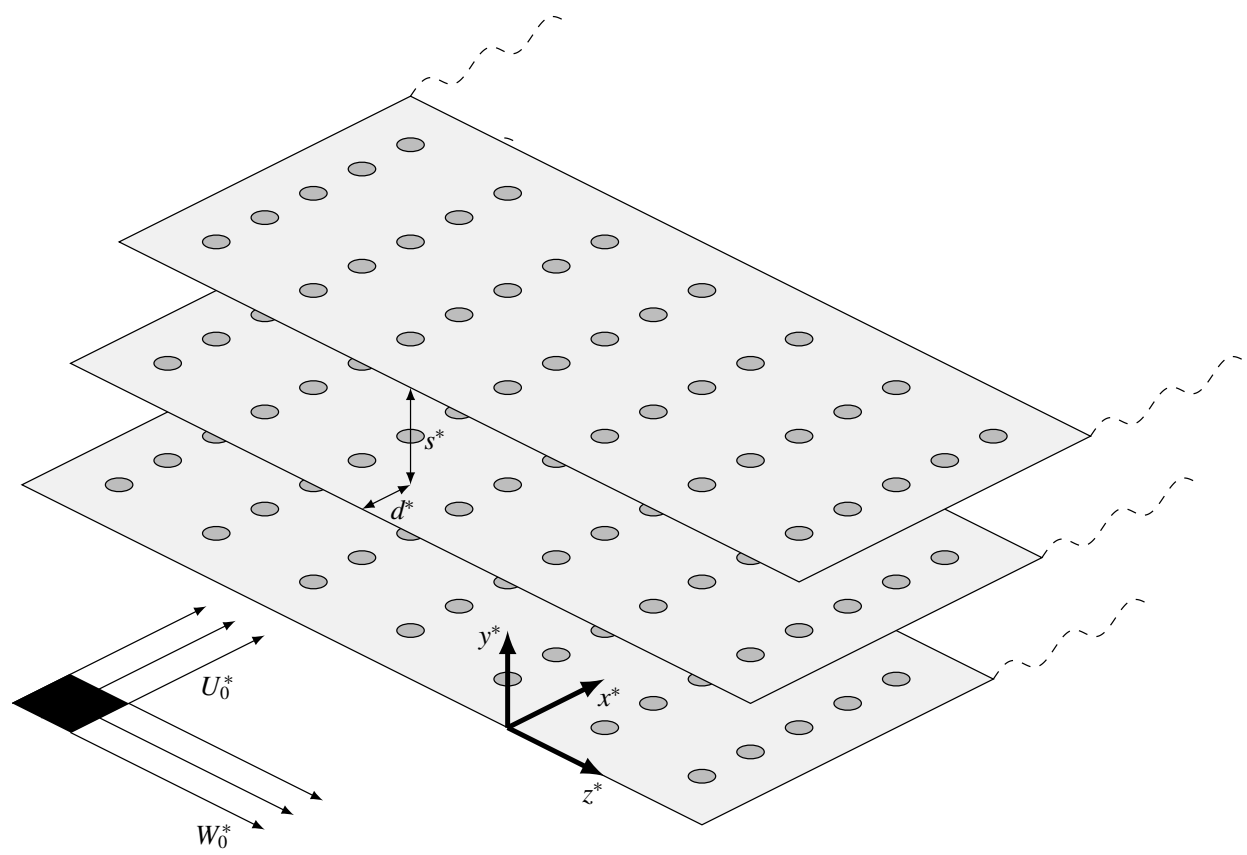

FIGURE 2. A three-dimensional view of the cascade in the rotated, dimensional $\left(x^{*}, y^{*}, z^{*}\right)$ coordinate system. The background velocities may have a spanwise component $W_{0}^{*}$. The complex boundaries are illustrated by the holes on each blade, which may represent compliance, porosity or impedance.

where $c_{0}$ is the isentropic speed of sound.

We suppose that the unsteady perturbation incident on the cascade takes the form

$$
\phi_{i}^{*}=\exp \left[\mathrm{i}\left(k_{x}^{*} x^{*}+k_{y}^{*} y^{*}+k_{z}^{*} z^{*}-\omega^{*} t^{*}\right)\right] .
$$

Dimensional variables are denoted with $*$ whereas non-dimensional variables have no annotation. Since the system is infinite in the spanwise direction, the scattered solution $\boldsymbol{u}^{*}$ must also have harmonic dependence in the $z^{*}$-direction. Accordingly, making the following convective transformation and non-dimensionalisations

$$
\begin{gathered}
\phi^{*}\left(x^{*}, y^{*}, z^{*}, t^{*}\right)=U_{0}^{*} b^{*} \phi(x, y) \exp \left[\mathrm{i} \omega\left(-\left(M_{0} / \beta\right)^{2} x+k_{z} z-t\right)\right], \\
d^{*}=b^{*} d, \quad s^{*}=b^{*} \frac{s}{\beta}, \quad \Delta=\sqrt{d^{2}+s^{2}}, \\
x^{*}=b^{*} x, \quad y^{*}=b^{*} \frac{y}{\beta}, \quad z^{*}=b^{*} z, \quad t^{*}=\frac{\omega}{\omega^{*}} t, \\
k_{x}^{*}=\frac{k_{x}-M_{0}^{2} \omega}{\beta^{2} b^{*}}, \quad k_{y}^{*}=\frac{\omega \beta k_{y}}{b^{*}}, \quad k_{z}^{*}=\frac{\omega k_{z}}{b^{*}}, \\
M_{0}=U_{0}^{*} / c_{0}, \quad \beta=\sqrt{1-M_{0}^{2}}, \\
\omega=\frac{b^{*}}{U_{0}^{*}}\left(\omega^{*}-W_{0}^{*} k_{z}^{*}\right), \quad W_{0}^{*}=U_{0}^{*} W_{0}, \\
w^{2}=\left(M_{0} / \beta^{2}\right)^{2}-\left(k_{z} / \beta\right)^{2}+2 k_{z} M_{0}^{4} W_{0}\left(1+W_{0} k_{z}\right) / \beta^{2},
\end{gathered}
$$


reduces (2.2) to

$$
\left(\frac{\partial^{2}}{\partial x^{2}}+\frac{\partial^{2}}{\partial y^{2}}+\omega^{2} w^{2}\right) \phi=0 .
$$

An alternative non-dimensionalisation is required when $U_{0}^{*}=0$, but this is a trivial modification. In terms of these new variables, the scattered pressure (2.1) becomes

$$
p^{*}=\rho_{0}^{*} U_{0}^{* 2} p \mathrm{e}^{-\mathrm{i} \omega t}, \quad p=-\frac{\partial}{\partial x}\left(\phi \mathrm{e}^{-\mathrm{i}\left(\omega / \beta^{2}\right) x}\right) \mathrm{e}^{\mathrm{i} \omega\left(x+k_{z} z\right)},
$$

where $p$ is the non-dimensional pressure, and the incident perturbation becomes

$$
\phi_{i}^{*}=U_{0}^{*} b^{*} \phi_{i}(x, y) \mathrm{e}^{\mathrm{i} \omega\left(-\left(M_{0} / \beta\right)^{2} x+k_{z} z-t\right)}, \quad \phi_{i}=\exp \left[\mathrm{i}\left(\left(k_{x} / \beta^{2}\right) x+\omega k_{y} y\right)\right] .
$$

\subsection{Boundary conditions}

We now introduce the boundary conditions for the problem. It is sufficient to specify the behaviour along $y=n s^{ \pm}$for $n \in \mathbb{Z}$. We use $\Delta_{n}[f]$ and $\Sigma_{n}[f]$ respectively to denote the difference and sum of $f$ either side of the $n$th blade or wake.

\subsubsection{Upstream boundary condition}

There may be no discontinuities upstream of the blade row. Consequently, we write

$$
\Delta_{n}[\phi](x)=0, \quad x<n d .
$$

\subsubsection{Blade surface boundary conditions}

We now introduce several possible blade surface boundary conditions that can be modelled with our solution. The types of blades we consider are the classical impermeable and rigid blade, a porous or compliant blade without background flow, a porous blade with background flow and a blade with a general impedance condition. All these blades admit closed-form, homogenised boundary conditions that are relatively simple to express. Moreover, all the boundary conditions take the same form. By solving for all possibly boundary condition simultaneously, we are able to model a range of scenarios of practical interest without modifying our solution. As we shall see later, the effect of modifying the boundary condition is to modify the kernel in the ensuing Wiener-Hopf analysis.

In this initial study, we focus on boundary conditions that are locally reacting so that the sound propagation at a given point on or inside the blade depends only on the acoustic pressure above and below that point. Accordingly, the surface impedance does not depend on the angle of the incident wave. For example, in the case of porous blades, we consider blades that can be characterised by lattices of unconnected, straight pores. In contrast, the sound field inside a connected pore will depend on the sound field in the neighbouring pores in addition to the sound pressure above and below the pore. The effects of non-locally reacting boundary conditions could, in principle, be considered by our solution if the corresponding boundary conditions can be homogenised for the infinitesimal blades that we consider. Additionally, we assume that the impedance is the same at all points on the blade e.g. the porosity is constant in both the chordwise and spanwise directions.

We now summarise the different types of boundary conditions that may be considered. 
Case 0

When the blade is rigid and impermeable, the no-flux condition is simply

$$
\boldsymbol{u}_{T} \cdot \boldsymbol{n}=0, \quad n d<x<n d+2, y=n s^{ \pm},
$$

where $\boldsymbol{n}$ is the normal vector directed away from the blade and $\boldsymbol{u}_{T}$ denotes the total (incident and scattered) velocity field. We sum the contributions of (2.8) either side of each blade to obtain

$$
\Sigma_{n}\left[\frac{\partial \phi}{\partial y}\right](x)=-2 w_{0} \exp \left[\mathrm{i}\left(\left(k_{x} / \beta^{2}\right)(n d+x)+\omega k_{y} n s\right)\right], \quad n d<x<n d+2,
$$

where $w_{0}=\mathrm{i} \omega k_{y}$ is the non-dimensional amplitude of the normal velocity of the incident perturbation on the 0th blade. This case has been considered in detail in previous research (Glegg 1999; Posson et al. 2010) and is therefore not considered further in the present work.

\section{Case I}

We now consider blades with perforations such as the circular apertures illustrated in figure 2. For a pressure load of frequency $\omega$, the Rayleigh conductivity $K_{R}$ relates the mass per unit time $(Q)$ flowing through an isolated aperture to the pressure difference either side of the aperture $\left(p^{+}-p^{-}\right)$by (Strutt 1870)

$$
K_{R}=\frac{\mathrm{i} \omega Q}{p^{+}-p^{-}}
$$

in our non-dimensional variables. The Rayleigh conductivity is a function of the aperture shape: $K_{R}=2 R$ for a circular aperture of radius $R$ in a zero-thickness plate. Recently, Brandão \& Schnitzer (2020) derived asymptotic expressions for the Rayleigh conductivity of a cylindrical orifice in a plate of finite thickness. These solutions also account for the viscous effects of a Stokes boundary later adjacent to the plate. A thorough discussion of the Rayleigh conductivity may be found in $\S 5.3 .1$ of Howe (1998).

The Rayleigh conductivity (2.10) can be deployed to derive an effective condition for a blade with multiple apertures through a homogenisation procedure. Suppose that the blade has $N$ perforations per unit area, each of conductivity $K_{R}$. Assuming that the acoustic wavelength is large compared to the aperture size, the pressure either side can be regarded as constant over the aperture length. If the apertures are sufficiently far apart, their conductivity can be measured as if they were in isolation. Therefore, (2.10) holds on the aperture scale and each aperture contributes a mass flux of $Q$. Consequently, the total mass flux per unit area is $N Q=N K_{R}\left(p^{+}-p^{-}\right) /(\mathrm{i} \omega)$ which must match with $v_{T}$ by continuity, where $v_{T}$ is the total velocity in the $y$-direction. Accordingly, we may simply write the effective conductivity $\tilde{K}_{R}$ for a plate with $N$ apertures per unit area with conductivity $K_{R}$ as $\tilde{K}_{R}=N K_{R}$. This argument has been made rigorous by Bendali et al. (2013). In the case of circular apertures of radius $R$, the effective conductivity becomes

$$
\tilde{K}_{R}=\frac{2 \alpha_{H}}{\pi R}
$$

for fractional open area $\alpha_{H}$. 
Substituting our flow variables into (2.10), we generalise the no-flux condition (2.8) and obtain

$$
v_{T}=\frac{\tilde{K}_{R}}{\mathrm{i} \omega} \Delta_{n}[p](x), \quad n d<x<n d+2, y=n s^{ \pm} .
$$

Summing the contributions either side of the blade in (2.11) yields

$$
\begin{aligned}
\Sigma_{n}\left[\frac{\partial \phi}{\partial y}\right](x)= & -2 w_{0} \exp \left[\mathrm{i}\left(\left(k_{x} / \beta^{2}\right)(n d+x)+\omega k_{y} n s\right)\right] \\
& -C_{I} \Delta_{n}[p](x), \quad n d<x<n d+2,
\end{aligned}
$$

for constant $C_{I}=-2 \tilde{K}_{R} /(\mathrm{i} \omega)$. In the absence of background flow, this condition becomes (subject to an alternative non-dimensionalisation)

$$
\begin{aligned}
\Sigma_{n}\left[\frac{\partial \phi}{\partial y}\right](x)= & -2 w_{0} \exp \left[\mathrm{i}\left(\left(k_{x} / \beta^{2}\right)(n d+x)+\omega k_{y} n s\right)\right] \\
& +C_{I} \Delta_{n}[\phi](x), \quad n d<x<n d+2 .
\end{aligned}
$$

This boundary condition has gained popularity as a tool for analysing the aerodynamic scattering of porous edges (Jaworski \& Peake 2013; Ayton 2016; Kisil \& Ayton 2018), but it is also valid in other scenarios: Leppington (1977) showed that (2.13) is equivalent to the boundary condition for an impermeable, compliant plate. Indeed, Crighton \& Leppington (1970) used (2.13) to analyse the scattering of aerodynamic sound by a semi-infinite compliant plate. In that study, the plate was modelled as possessing inertia, but negligible elastic resistance to deformation. Consequently, the pressure difference across the compliant plate was proportional to the specific mass of the plate multiplied by the acceleration so that, in the notation of the present work, the constant $C_{I}$ would instead become $C_{I}=-2 / m$ where $m$ is the (non-dimensional) mass of the plate per unit area.

Case II

We now consider the case where the plates are porous and embedded in a mean flow. The mean flow has a significant effect on the Rayleigh conductivity for an aperture, and analytic expressions for $K_{R}$ are generally intractable. However, Howe, Scott \& Sipcic (1996) showed that, for a circular aperture in a grazing flow, the Rayleigh conductivity is

$$
K_{R}(\omega)=R \int_{S} v(\xi, \eta, \omega) \mathrm{d} \xi \mathrm{d} \eta
$$

where the integral is taken over the aperture area $\left(\xi^{2}+\eta^{2}<R^{2}\right)$. The function $v(\xi, \eta, \omega)$ corresponds to the displacement of fluid at the aperture interface and must be found as the solution to an integral equation defined in Howe et al. (1996). This equation must generally be solved numerically and a range of values for $K_{R}(\omega)$ are recorded in table 2 of Howe et al. (1996). Unlike case I boundary conditions, the conductivity may be complex so it is convenient to separate the complex conductivity into real and imaginary parts

$$
K_{R}(\omega)=2 R\left(\Gamma_{R}(\omega)-\mathrm{i} \Delta_{R}(\omega)\right),
$$


where $\Gamma_{R}(\omega)$ and $\Delta_{R}(\omega)$ are real valued functions of frequency. Using a similar argument to the previous section, we may write the effective compliance for a perforated plate as $\tilde{K}_{R}=N K_{R}$. The no-flux condition (2.8) now generalises to

$$
\begin{aligned}
\Sigma_{n}\left[\frac{\partial \phi}{\partial y}\right](x)= & -2 w_{0} \exp \left[\mathrm{i}\left(\left(k_{x} / \beta^{2}\right)(n d+x)+\omega k_{y} n s\right)\right] \\
& +C_{I I}\left(\mathrm{i}\left(\omega / \beta^{2}\right) \Delta_{n}[\phi](x)-\Delta_{n}\left[\phi_{x}\right](x)\right), \quad n d<x<n d+2,
\end{aligned}
$$

where again $C_{I I}=2 \tilde{K}_{R}(\omega) /(\mathrm{i} \omega)$ is a constant.

\section{Case III}

We may also consider the effects of an impedance boundary condition. In the presence of background flow, the impedance boundary condition is given by Myers (1980)

$$
\boldsymbol{u}_{T} \cdot \boldsymbol{n}=\left(\frac{\partial}{\partial t}+\boldsymbol{U}_{0} \cdot \nabla-\boldsymbol{n} \cdot\left(\boldsymbol{n} \cdot \nabla \boldsymbol{U}_{0}\right)\right) \frac{p}{\mathrm{i} \omega Z},
$$

where $\boldsymbol{n}$ is now directed into the blade. The real part of the impedance $Z$ is termed the acoustic resistance and represents the energy transfer of the blade: if $\operatorname{Re}[Z]>0$ then the blades absorb energy whereas if $\operatorname{Re}[Z]<0$ then the blades produce energy. Since the flow is uniform, this condition applied on the upper and lower surfaces of the blades becomes, in terms of the present variables,

$$
v_{T}=\mp\left(\frac{\partial}{\partial x}-\mathrm{i} \omega\right) \frac{p}{\mathrm{i} \omega Z} .
$$

We now sum the upper and lower components of this impedance condition to obtain a condition on the sum of the velocity either side of the blade

$$
\begin{aligned}
\Sigma_{n}\left[\frac{\partial \phi}{\partial y}\right](x)= & -2 w_{0} \exp \left[\mathrm{i}\left(\left(k_{x} / \beta^{2}\right)(n d+x)+\omega k_{y} n s\right)\right] \\
& +C_{I I I}\left(-\frac{\omega^{2}}{\beta^{4}} \Delta_{n}[\phi](x)-\frac{2 \mathrm{i} \omega}{\beta^{2}} \Delta_{n}\left[\phi_{x}\right](x)+\Delta_{n}\left[\phi_{x, x}\right](x)\right),
\end{aligned}
$$

where $C_{I I I}=1 /(\mathrm{i} \omega Z)$.

The presence of higher-order derivatives requires further regularity at the blades' edges. Since the blades are fixed (and are only locally reacting), we additionally enforce that $\Delta_{n}\left[\phi_{x}\right](0)$ is bounded.

Summary of blade surface boundary conditions

We may characterise all the modified boundary conditions (2.13), (2.15) and (2.16) in the general form

$$
\begin{aligned}
& \Sigma_{n}\left[\frac{\partial \phi}{\partial y}\right](x)=-2 w_{0} \exp \left[\mathrm{i}\left(\left(k_{x} / \beta^{2}\right)(n d+x)+\omega k_{y} n s\right)\right] \\
& \quad+\mu_{0} \Delta_{n}[\phi](x)+\mu_{1} \Delta_{n}\left[\phi_{x}\right](x)+\mu_{2} \Delta_{n}\left[\phi_{x, x}\right](x), \quad n d<x<n d+2,
\end{aligned}
$$

where the $\mu_{n}$ are summarised in table 1 for the different boundary conditions. Furthermore, in the present analysis we do not allow any added mass generated in the blades and enforce that there is no jump in the normal velocity either side of the plate. Accordingly, we may write

$$
\Delta_{n}\left[\frac{\partial \phi}{\partial y}\right](x)=0, \quad n d<x<n d+2 .
$$




$\begin{array}{lcccc}\text { Case } & \text { Model } & \mu_{0} & \mu_{1} & \mu_{2} \\ \begin{array}{l}\text { Case 0 } \\ {[1,2]}\end{array} & \text { Rigid and impermeable } & 0 & 0 & 0 \\ \begin{array}{l}\text { Case I } \\ {[3,4,5,6]}\end{array} & \begin{array}{c}\text { Porous, compliant } \\ \text { (no background flow) }\end{array} & C_{I} & 0 & 0 \\ \begin{array}{l}\text { Case II } \\ {[7]}\end{array} & \text { (with background flow) } & \mathrm{i}\left(\omega / \beta^{2}\right) C_{I I} & -C_{I I} & 0 \\ \text { Case III } & \text { Impedance } & -\left(\omega^{2} / \beta^{4}\right) C_{I I I} & -\left(2 \mathrm{i} \omega / \beta^{2}\right) C_{I I I} & C_{I I I}\end{array}$

TABLE 1. Summary of possible boundary conditions and corresponding $\mu_{0}, \mu_{1}$ and $\mu_{2}$ values for (2.17). The references highlight relevant papers, although only [1,2] consider cascade geometries and are restricted to impermeable and rigid boundaries. The reference numbers correspond to [1] (Glegg 1999), [2] (Posson et al. 2010), [3] (Leppington 1977), [4] (Howe 1998), [5] (Jaworski \& Peake 2013), [6] (Kisil \& Ayton 2018), [7] (Howe et al. 1996), [8] (Myers 1980) and [9] (Brambley 2009).

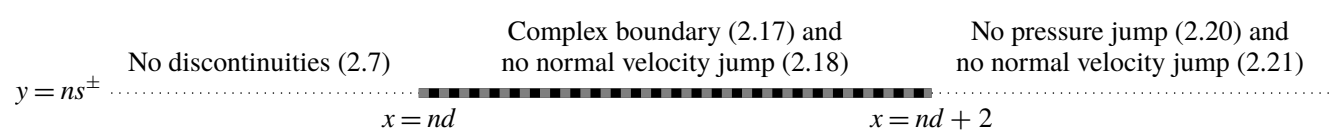

FIGURE 3. Schematic illustrating where each boundary condition is applied.

\subsubsection{Downstream boundary conditions}

Downstream, we require the pressure jump across the wake to vanish:

$$
\Delta_{n}[p](x)=0, \quad x>2+n d .
$$

By employing the pressure definition (2.5) and integrating with respect to $x$, we may write the above condition as

$$
\Delta_{n}[\phi](x)=2 \pi \mathrm{i} P \exp \left[\mathrm{i}\left(\omega / \beta^{2}\right) x\right], \quad x>n d+2,
$$

where $P$ is a constant of integration that will be specified by enforcing the Kutta condition.

Additionally, the normal velocity across the wake must vanish, i.e.

$$
\Delta_{n}\left[\frac{\partial \phi}{\partial y}\right](x)=0, \quad x>n d+2 .
$$

\subsubsection{Summary of full boundary conditions}

All in all, we have five boundary conditions. In the upstream region we do not permit any discontinuities (2.7). Along each blade we have a relation for the sum of normal velocities either side of the blade (2.17), and do not permit a jump in normal velocity across the blade (2.18). Finally, across the wake we do not permit a jump in pressure (2.20) or normal velocity (2.21). The boundary conditions are illustrated in figure 3 . This completes the description of the mathematical model. 


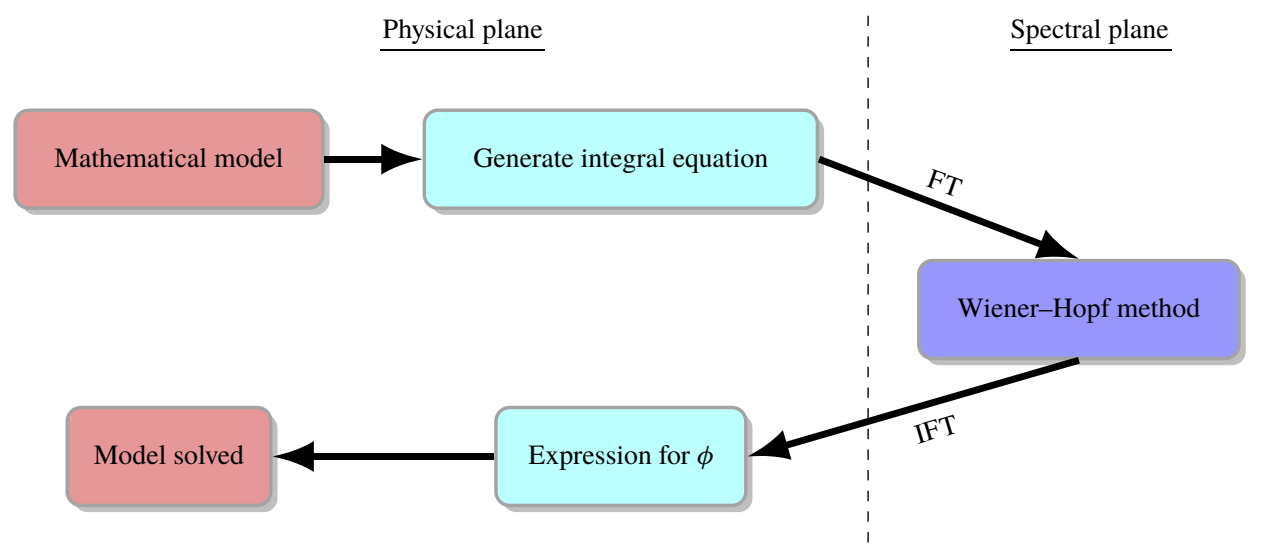

FIGURE 4. Schematic diagram illustrating the solution method. The abbreviations 'FT' and 'IFT' stand for 'Fourier transform' and 'Inverse Fourier transform' respectively.

\section{Solution}

We now present the mathematical solution to the Helmholtz equation (2.4) subject to the boundary conditions (2.7), (2.17), (2.18), (2.20) and (2.21). For clarity, we present a 'road map' of the solution in figure 4. Further details of the solution procedure may be found in Baddoo \& Ayton (2020).

As is typical in cascade acoustics problems we employ integral transforms to obtain a solution that is uniformly valid throughout the entire domain (Peake 1992; Glegg 1999; Posson et al. 2010). However, $\phi$ is discontinuous across each blade and wake in the $y$-direction. Therefore, $\partial \phi / \partial y$ possesses non-integrable singularities thus preventing the application of a Fourier transform. Consequently, we must regularise the derivatives of $\phi$ to remove these non-integrable singularities. To this end, we introduce introduce generalised derivatives (Lighthill 1958) and write

$$
\frac{\partial^{2} \phi}{\partial y^{2}}=\frac{\tilde{\partial}^{2} \phi}{\tilde{\partial} y^{2}}-\sum_{n=-\infty}^{\infty} \Delta_{n}[\phi](x) \delta^{\prime}(y-n s)-\sum_{n=-\infty}^{\infty} \Delta_{n}\left[\frac{\partial \phi}{\partial y}\right](x) \delta(y-n s),
$$

where $\tilde{\partial}$ represents the partial derivative with discontinuities removed, and $\delta$ here represents the Dirac delta function. The third term on the right side of (3.1) vanishes because there is zero jump in normal velocity across the blade (2.18) and wake (2.21).

The scattered solution must obey the same quasi-periodicity relation as the incident field (2.3). Consequently, the scattered acoustic potential function in the entire plane may be reduced to that of a single channel in the domain by writing

$$
\phi(x+n d, y+n s)=\phi(x, y) \mathrm{e}^{\mathrm{i} n \sigma^{\prime}},
$$

where the inter-blade phase angle for $\phi$ is $\sigma^{\prime}=\left(k_{x} / \beta^{2}\right) d+\omega k_{y} s$. For example, we may use (3.2) to reduce the jumps in potential to

$$
\Delta_{n}[\phi](x)=\Delta_{0}[\phi](x-n d) \mathrm{e}^{\mathrm{i} n \sigma^{\prime}} .
$$


Substituting (3.1) into the Helmholtz equation (2.4) and applying the inter-blade phase angle relation (3.3) yields

$$
\frac{\tilde{\partial}^{2} \phi}{\tilde{\partial} x^{2}}+\frac{\tilde{\partial}^{2} \phi}{\tilde{\partial} y^{2}}+\omega^{2} w^{2} \phi=\sum_{n=-\infty}^{\infty} \Delta_{0}[\phi]\left(x-n d_{\phi}\right) \delta^{\prime}\left(y-n s_{\phi}\right) \mathrm{e}^{\mathrm{i} n \sigma^{\prime}} .
$$

We define the Fourier integral transform and its inverse as

$$
\begin{gathered}
F(\gamma, \eta)=\frac{1}{(2 \pi)^{2}} \int_{-\infty}^{\infty} \int_{-\infty}^{\infty} f(x, y) \mathrm{e}^{\mathrm{i} \gamma x+\mathrm{i} \eta y} \mathrm{~d} x \mathrm{~d} y, \\
f(x, y)=\int_{-\infty}^{\infty} \int_{-\infty}^{\infty} F(\gamma, \eta) \mathrm{e}^{-\mathrm{i} \gamma x-\mathrm{i} \eta y} \mathrm{~d} \gamma \mathrm{d} \eta .
\end{gathered}
$$

Applying the transform to the left-hand side of (3.4) yields

$$
\left(-\gamma^{2}-\eta^{2}+\omega^{2} w^{2}\right) \Phi(\gamma, \eta)=\frac{1}{2 \pi \mathrm{i}} \sum_{n=-\infty}^{\infty} \eta D(\gamma) \mathrm{e}^{\mathrm{i} n\left(\sigma^{\prime}+\gamma d_{\phi}+\eta s_{\phi}\right)},
$$

where $\Phi$ is the Fourier transform of $\phi$. The problem is now to find $D(\gamma)$ which represents the Fourier transform of the jump in acoustic potential either side of the blade and wake

$$
D(\gamma)=\frac{1}{2 \pi} \int_{0}^{\infty} \Delta_{0}[\phi](x) \mathrm{e}^{\mathrm{i} \gamma x} \mathrm{~d} x .
$$

We invert the Fourier transform to obtain an expression for the acoustic potential in terms of $D$

$$
\phi(x, y)=\frac{1}{2 \pi \mathrm{i}} \int_{-\infty}^{\infty} \int_{-\infty}^{\infty} \frac{\mathrm{e}^{-\mathrm{i} \gamma x-\mathrm{i} \eta y}}{\omega^{2} w^{2}-\eta^{2}-\gamma^{2}} \cdot \sum_{n=-\infty}^{\infty} \eta D(\gamma) \mathrm{e}^{\mathrm{i} n\left(\sigma^{\prime}+\gamma d+\eta s\right)} \mathrm{d} \gamma \mathrm{d} \eta .
$$

The $\eta$ integral may be performed by closing the contour of integration in an appropriate upper or lower half-plane to obtain

$$
\phi(x, y)=-\frac{1}{2} \int_{-\infty}^{\infty} \sum_{n=-\infty}^{\infty} D(\gamma) \operatorname{sgn}(n s-y) \mathrm{e}^{\mathrm{i} n\left(\sigma^{\prime}+\gamma d\right)+\mathrm{i} \zeta|n s-y|} \mathrm{e}^{-\mathrm{i} \gamma x} \mathrm{~d} \gamma,
$$

where $\zeta=\sqrt{\omega^{2} w^{2}-\gamma^{2}}$. The branch cut is defined so that $\operatorname{Im}[\zeta]>0$ when $\gamma$ is in a strip for the Wiener-Hopf method.

To obtain an equation for the unknown $D$ we must apply the relevant boundary conditions. We first differentiate (3.7) with respect to $y$ and consider the limits $y \rightarrow 0^{ \pm}$. Summing the contributions from each of these limits yields the integral equation

$$
\Sigma_{0}\left[\frac{\partial \phi}{\partial y}\right](x)=4 \pi \int_{-\infty}^{\infty} D(\gamma) j(\gamma) \mathrm{e}^{-\mathrm{i} \gamma x} \mathrm{~d} \gamma,
$$

where

$$
j(\gamma)=\frac{\mathrm{i} \zeta}{4 \pi} \sum_{n \in \mathbb{Z}} \mathrm{e}^{\mathrm{i} n\left(\sigma^{\prime}+\gamma d\right)+\mathrm{i} \zeta|n s|}=\frac{\zeta}{4 \pi} \cdot \frac{\sin (\zeta s)}{\cos (\zeta s)-\cos \left(\gamma d+\sigma^{\prime}\right)} .
$$


We now solve (3.8) subject to the remaining boundary conditions applied on $y=0$

$$
\begin{gathered}
\Delta_{0}[\phi](x)=0, \quad x<0, \\
\Sigma_{0}\left[\frac{\partial \phi}{\partial y}\right](x)=\mu_{0} \Delta_{0}[\phi](x)+\mu_{1} \Delta_{0}\left[\phi_{x}\right](x)+\mu_{2} \Delta_{0}\left[\phi_{x, x}\right](x) \\
-2 w_{0} \exp \left[\mathrm{i}\left(k_{x} / \beta^{2}\right) x\right], \quad 0<x<2, \\
\Delta_{0}[\phi](x)=2 \pi \mathrm{i} P \exp \left[\mathrm{i}\left(\omega / \beta^{2}\right) x\right], \quad x>2 .
\end{gathered}
$$

The system $((3.8),(3.10 a),(3.10 b),(3.10 c))$ represents an integral equation subject to mixed value boundary conditions. We solve this system via the Wiener-Hopf method as detailed in appendix A. The solution for $D$ is given by

$$
\begin{aligned}
D(\gamma)= & \frac{w_{0}}{(2 \pi)^{2} \mathrm{i}\left(\gamma+k_{x} / \beta^{2}\right) K_{-}\left(-k_{x} / \beta^{2}\right) K_{+}(\gamma)} \\
& +\frac{w_{0}\left(\omega-k_{x}\right) \mathrm{e}^{2 \mathrm{i}\left(\gamma+k_{x} / \beta^{2}\right)}}{(2 \pi \beta)^{2} \mathrm{i}\left(\gamma+k_{x} / \beta^{2}\right)\left(\gamma+\omega / \beta^{2}\right) K_{+}\left(-k_{x} / \beta^{2}\right) K_{-}(\gamma)} \\
& -\sum_{n=0}^{\infty} \frac{\left(\mathcal{A}_{n}+\mathcal{C}_{n}\right) \mathrm{e}^{2 \mathrm{i}\left(\gamma-\theta_{n}^{-}\right)}}{\mathrm{i}\left(\gamma+\omega / \beta^{2}\right)\left(\gamma-\theta_{n}^{-}\right)} \cdot \frac{K_{-}\left(\theta_{n}^{-}\right)}{K_{-}(\gamma)}-\sum_{n=0}^{\infty} \frac{\mathcal{B}_{n}}{\gamma-\theta_{n}^{+}} \cdot \frac{K_{+}\left(\theta_{n}^{+}\right)}{K_{+}(\gamma)},
\end{aligned}
$$

where all new variables are defined in appendix A. Note that the solution is identical to that for the type 0 cascade (Glegg 1999), except the original Wiener-Hopf kernel $j$ is now replaced with the modified kernel $K$. This original kernel is recovered when $\mu_{1}=\mu_{2}=\mu_{3}=0$ and the solution reduces to that derived by Glegg (1999).

\subsection{Inversion of Fourier transform}

We now invert the Fourier transform of the previous section to obtain the acoustic potential function. Since $D$ is now known, the Fourier inversion integral in (3.7) can now be computed. Similarly to the analysis in Posson et al. (2010), the inversion is performed by splitting the physical domain into five regions as illustrated in figure 5 . Each solution is simply a sum of exponential functions. The details can be found in appendix $\mathrm{C}$ and the final results are stated below. All undefined functions are defined in appendices $\mathrm{A}$ and $\mathrm{C}$.

\subsubsection{Upstream region (I)}

In the upstream region,

$$
\phi(x, y)=2 \pi \mathrm{i} \sum_{m=-\infty}^{\infty} D^{(1,3)}\left(\lambda_{m}^{+}\right) A^{r}\left(x, y ; \lambda_{m}^{+}\right) .
$$

\subsubsection{Inter-blade upstream region (II)}

In the inter-blade upstream region,

$$
\begin{aligned}
\phi(x, y)= & -2 \pi \sum_{n=0}^{\infty} \frac{\mathcal{A}_{n}+\mathcal{C}_{n}}{\theta_{n}^{-}+\omega / \beta^{2}} A_{d}\left(x, y ; \theta_{n}^{-}\right)+2 \pi \mathrm{i} \sum_{n=0}^{\infty} \mathcal{B}_{n} A_{d}\left(x, y ; \theta_{n}^{+}\right) \\
& -\frac{A_{d}\left(x, y ;-k_{x} / \beta^{2}\right)}{K\left(-k_{x} / \beta^{2}\right)} \frac{w_{0}}{2 \pi}+2 \pi \mathrm{i} \sum_{m=-\infty}^{\infty} D^{(1,3)}\left(\lambda_{m}^{+}\right) A_{u}^{r}\left(x, y ; \lambda_{m}^{+}\right) .
\end{aligned}
$$




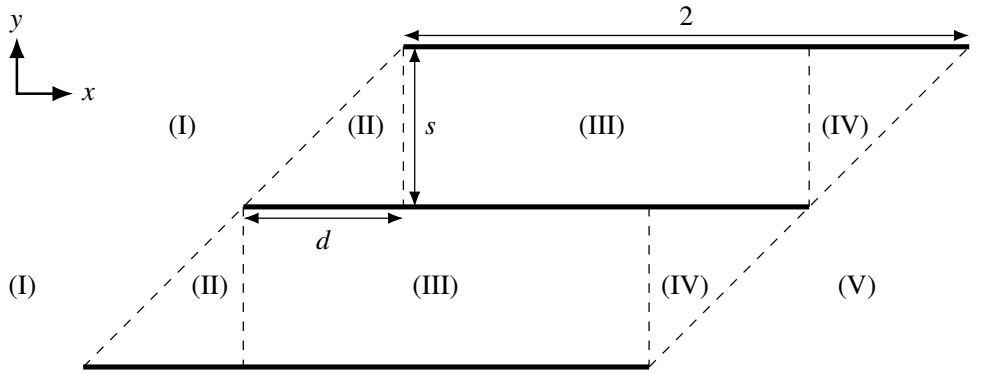

FIGURE 5. Diagram indicating the different regions in the $(x, y)$-plane which require different areas of contour integration in the Fourier inversion.

\subsubsection{Inter-blade inner region (III)}

In the inter-blade inner region,

$$
\begin{aligned}
\phi(x, y)= & -2 \pi \sum_{n=0}^{\infty} \frac{\mathcal{A}_{n}+\mathcal{C}_{n}}{\theta_{n}^{-}+\omega / \beta^{2}} A\left(x, y ; \theta_{n}^{-}\right)+2 \pi \mathrm{i} \sum_{n=0}^{\infty} \mathcal{B}_{n} A\left(\theta_{n}^{+}, x, y\right) \\
& -\frac{A\left(x, y ;-k_{x} / \beta^{2}\right)}{K\left(-k_{x} / \beta^{2}\right)} \frac{w_{0}}{2 \pi} .
\end{aligned}
$$

\subsubsection{Inter-blade downstream region (IV)}

In the inter-blade downstream region,

$$
\begin{aligned}
\phi(x, y)= & -2 \pi \sum_{n=0}^{\infty} \frac{\mathcal{A}_{n}+\mathcal{C}_{n}}{\theta_{n}^{-}+\omega / \beta^{2}} A_{u}\left(x, y ; \theta_{n}^{-}\right)+2 \pi \mathrm{i} \sum_{n=0}^{\infty} \mathcal{B}_{n} A_{u}\left(x, y ; \theta_{n}^{+}\right) \\
& -\frac{A_{u}\left(x, y ;-k_{x} / \beta^{2}\right)}{K\left(-k_{x} / \beta^{2}\right)} \frac{w_{0}}{2 \pi} \\
& -2 \pi \mathrm{i} \sum_{m=-\infty}^{\infty} D^{(2,4)}\left(\lambda_{m}^{-}\right) A_{d}^{r}\left(x, y ; \lambda_{m}^{-}\right)+2 \pi \mathrm{i} P A_{d}\left(x, y ;-\omega / \beta^{2}\right) .
\end{aligned}
$$

\subsubsection{Downstream region $(V)$}

In the downstream region,

$$
\phi(x, y)=-2 \pi \mathrm{i} \sum_{m=-\infty}^{\infty} D^{(2,4)}\left(\lambda_{m}^{-}\right) A^{r}\left(x, y ; \lambda_{m}^{-}\right)+2 \pi \mathrm{i} P A\left(x, y ;-\omega / \beta^{2}\right) .
$$

\section{Results}

We now use the analytic solution derived in $\S 3.1$ to explore the aeroacoustic performance of a blade row with modified boundary conditions. In particular, we focus on the role of porosity due to its potential to attenuate sound, as seen previously in Jaworski \& Peake (2013) for trailing-edge scattering. Porous blades in flow are represented by the case II boundary condition in the present nomenclature. The results in the present research also show significant sound reductions for modest changes in porosity. We argue that this is attributed to the strong effect of porosity on the duct 


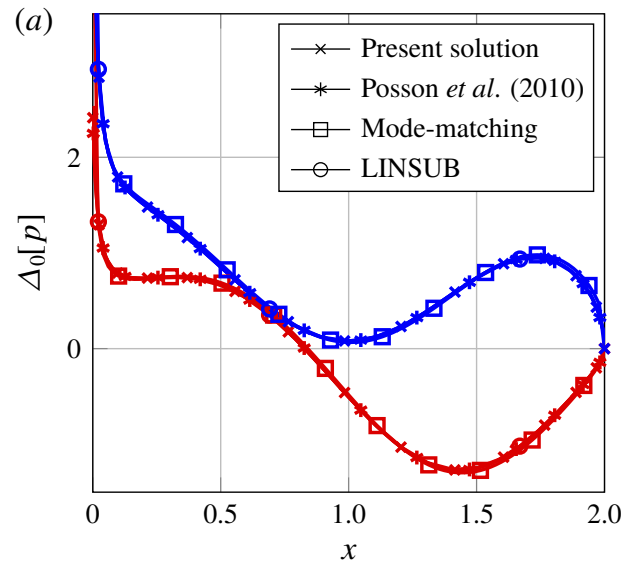

Case A (b)

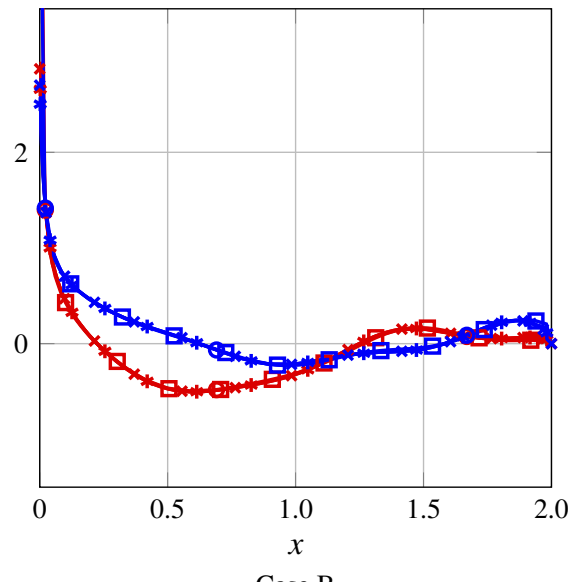

Case B

FIgURE 6. Comparisons of surface pressure jump for flat plates for case 0 boundary conditions and geometries defined in table 2. The real and imaginary parts (blue and red respectively) of the pressure jump are compared to three alternative solutions: a Wiener-Hopf method (Posson et al. 2010), a mode-matching technique (Bouley et al. 2017) and a numerical method (Hall 1997).

modes and unsteady loading: in cascade configurations, the blade loading changes the upstream and downstream flows and therefore influences the intensity of the scattered sound.

Although the solution is formally analytic, there are two steps that must be handled numerically. First, the linear system comprised of (A 33) and (A 36) must be solved. Second, the zeros of the meromorphic kernel $K$ must be located in the complex plane. For large zeros $\left(\left|\theta_{j}\right| \gg 1\right)$, the asymptotic approximations in $\S$ B.2 can reliably be used as initial guesses for an iterative Newton scheme. For other zeros $\left(\left|\theta_{j}\right| \sim 1\right)$, we use a modified version of the Delves \& Lyness (1967) algorithm to ensure that all the zeros in a prescribed region are found. The relevant integrations are computed using the trapezoidal rule, which converges geometrically for integrals of meromorphic functions over ellipses (Trefethen \& Weideman 2014). This rapid numerical implementation allows all relevant aeroacoustic quantities to be evaluated in a fraction of a second using the code publicised in the introduction.

\subsection{Validation}

We first present a comparison to three previous solutions for cascades of impermeable and rigid blades in figure 6. First, we compare our results to a solution exploiting the Wiener-Hopf method (Posson et al. 2010). Second, we compare to a quasi-numerical a mode-matching technique (Bouley et al. 2017), and third, we compare to a fully numerical method (Hall 1997). The solutions show excellent agreement - in fact our case 0 solution is mathematically equivalent to the of Posson et al. (2010). It is worth noting at this point that our solution satisfies the Kutta condition, as indicated by the pressure jump vanishing at the trailing edge.

\subsection{Duct mode analysis}

The cascade may be divided into five regions as illustrated in figure 5. Since we only consider the case where the blades are overlapping, the inter-blade inner region (called 
region III) is bounded by adjacent blades and therefore may be viewed as a duct. The solution in the duct is matched to the upstream and downstream regions by virtue of the inter-blade upstream region (II) and the inter-blade downstream region (IV). The duct region is therefore essential in establishing the relationship between the upstream and downstream regions, and controls the unsteady lift and sound power output. We now explore the behaviour of the solution in the duct in order to understand the effects of blade porosity.

The acoustic potential in the duct may be expanded into a sum of exponential functions whose modes are the 'duct modes' (Glegg \& Devenport 2017). For type 0 blades, the duct has rigid, impermeable walls and the modes are given by the standard formula

$$
\hat{\theta}_{n}^{ \pm}= \pm \sqrt{\omega^{2} w^{2}-\left(\frac{n \pi}{s}\right)^{2}}
$$

where we have used $\hat{\imath}$ to indicate that this solution is valid for case $0\left(C_{I I}=0\right)$. Conversely, there is no simple expression for the duct modes when the blades are porous; for porosity constant $C_{I I}$ the duct modes satisfy the transcendental equation

$$
\zeta\left(\theta_{n}^{ \pm}\right) \sin \left(s \zeta\left(\theta_{n}^{ \pm}\right)\right)=\mathrm{i} C_{I I}\left(\omega / \beta^{2}+\theta_{n}^{ \pm}\right)\left(\cos \left(s \zeta\left(\theta_{n}^{ \pm}\right)\right)-\cos \left(d \theta_{n}^{ \pm}+\sigma^{\prime}\right)\right) .
$$

The solutions for large $\theta_{n}^{ \pm}$are available via the asymptotic analysis presented in $\S$ B.2, but otherwise the solutions must generally be found with a numerical root finding algorithm (Delves \& Lyness 1967).

It is straightforward to see from (4.1) that the $n$th case 0 duct mode is pure imaginary when $\omega w<n \pi / s$ and pure real when $\omega w>n \pi / s$. These conditions correspond to the duct mode being cut-off or cut-on. However, inspection of (4.2) reveals that for an arbitrary finite porosity constant $C_{I I}$ (but not pure imaginary), it is impossible for the duct modes to be cut-on. This is readily seen by noting that there are no real solutions to (4.2). If a real root did exist, then the left hand side would be pure real. However, in that case the right hand side has a non-zero imaginary part, and we have a contradiction. Consequently, for any non-imaginary porosity coefficients, the duct modes are always complex and never pure real. Accordingly, blade porosity effects a reduction in the magnitude of the pressure field in the inter-blade inner region which, when matched to the upstream and downstream regions, results in a reduction in the far-field scattered sound.

The dependence of the duct modes on blade porosity is illustrated in figure 7 for two frequencies. There are no acoustic modes cut-on in figure 7(a), whereas there are two acoustic modes cut-on in figure $7(b)$. Modes located in the upper or lower half plane (denoted by $\mathcal{L}^{ \pm}$) are propagating in the upstream and downstream directions respectively. We consider a range of arguments for the porosity coefficient to represent a phase difference between the pressure jump and normal velocity, which is permitted due to the presence of the background flow (Howe et al. 1996). Evidently, the relationship between the duct modes is highly complex, although some general trends may be observed. In compliance with the argument in the preceding paragraph, all values of porosity (except pure imaginary) perturb the cut-on duct modes away from the real line. For zero porosity, the duct modes are located at the 


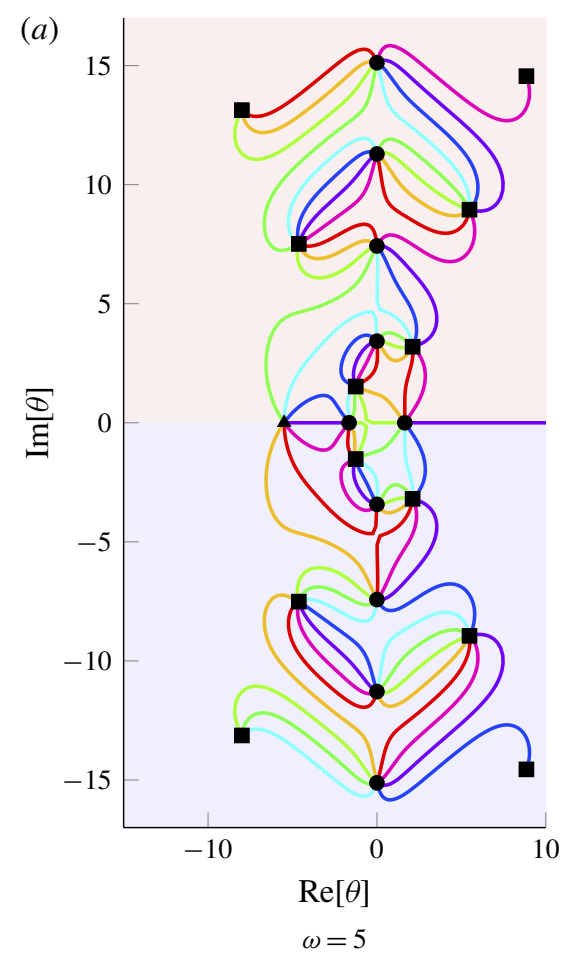

(b)

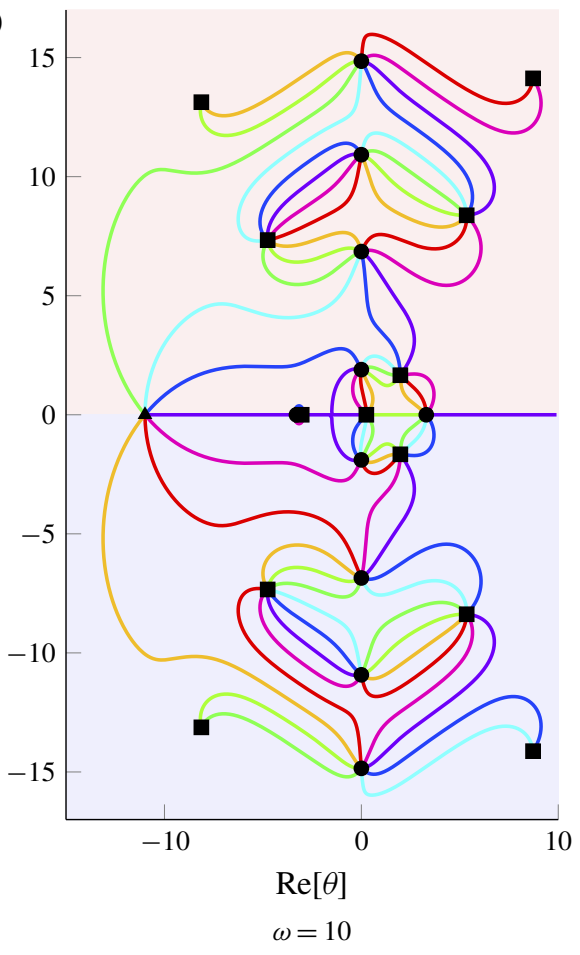

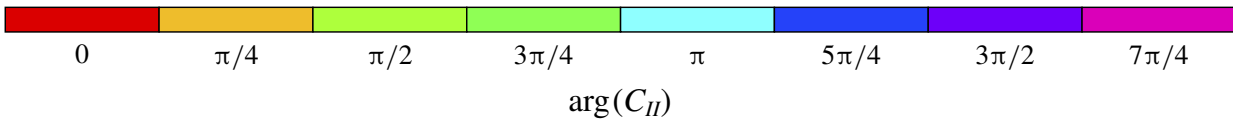

FIGURE 7. The trajectories of the duct modes for a range of (complex) porosity coefficients for case $\mathrm{C}$ described in table 2, with $k_{x}=4$. Along each line, the phase of $C_{I I}$ is held constant while its magnitude is increased from 0 to $\infty$. For example, the duct modes corresponding to real and positive values of $C_{I I}$ are illustrated in red. The duct modes for case 0 blades (i.e. $C_{I I}=0$ ) are denoted by $\bullet$, the acoustic modes, $\lambda_{m}$, are labelled denoted by $\boldsymbol{\square}$ and the convected mode $\left(-\omega / \beta^{2}\right)$ is denoted by $\boldsymbol{\Delta}$. Accordingly, each trajectory begins at a $\bullet$ and ends at either a $\boldsymbol{\square}$ or a $\boldsymbol{\Lambda}$. The upper half-plane $\mathcal{L}^{+}$is shaded in red and the lower half-plane $\mathcal{L}^{-}$is shaded in blue.

rigid duct modes, $\hat{\theta}_{n}^{ \pm}$. As the porosity is increased, the duct modes follow a path that generally ends at either the convected mode $\left(-\omega / \beta^{2}\right)$ or an acoustic mode $\left(\lambda_{n}^{ \pm}\right)$.

It is instructive to inspect the asymptotic forms of the duct modes for small and large values of porosity. For small Rayleigh conductivity $\left(\left|C_{I I}\right| \ll 1, \arg \left(C_{I I}\right) \neq \pm \pi / 2\right)$, the roots are a small perturbation away from the case 0 duct modes

$$
\theta_{n}^{ \pm}=\hat{\theta}_{n}^{ \pm}-C_{I I} \frac{\mathrm{i}\left(\omega / \beta^{2}+\hat{\theta}_{n}^{ \pm}\right)}{s\left(1+\delta_{0, n}\right) \hat{\theta}_{n}^{ \pm}}\left(1-(-1)^{n} \cos \left(d \hat{\theta}_{n}^{ \pm}+\sigma^{\prime}\right)\right)+o\left(C_{I I}\right)
$$

Conversely, for large Rayleigh conductivity $\left(\left|C_{I I}\right| \gg 1, \arg \left(C_{I I}\right) \neq \pm \pi / 2\right)$, the duct modes are a small perturbation away from either the hydrodynamic mode or the 

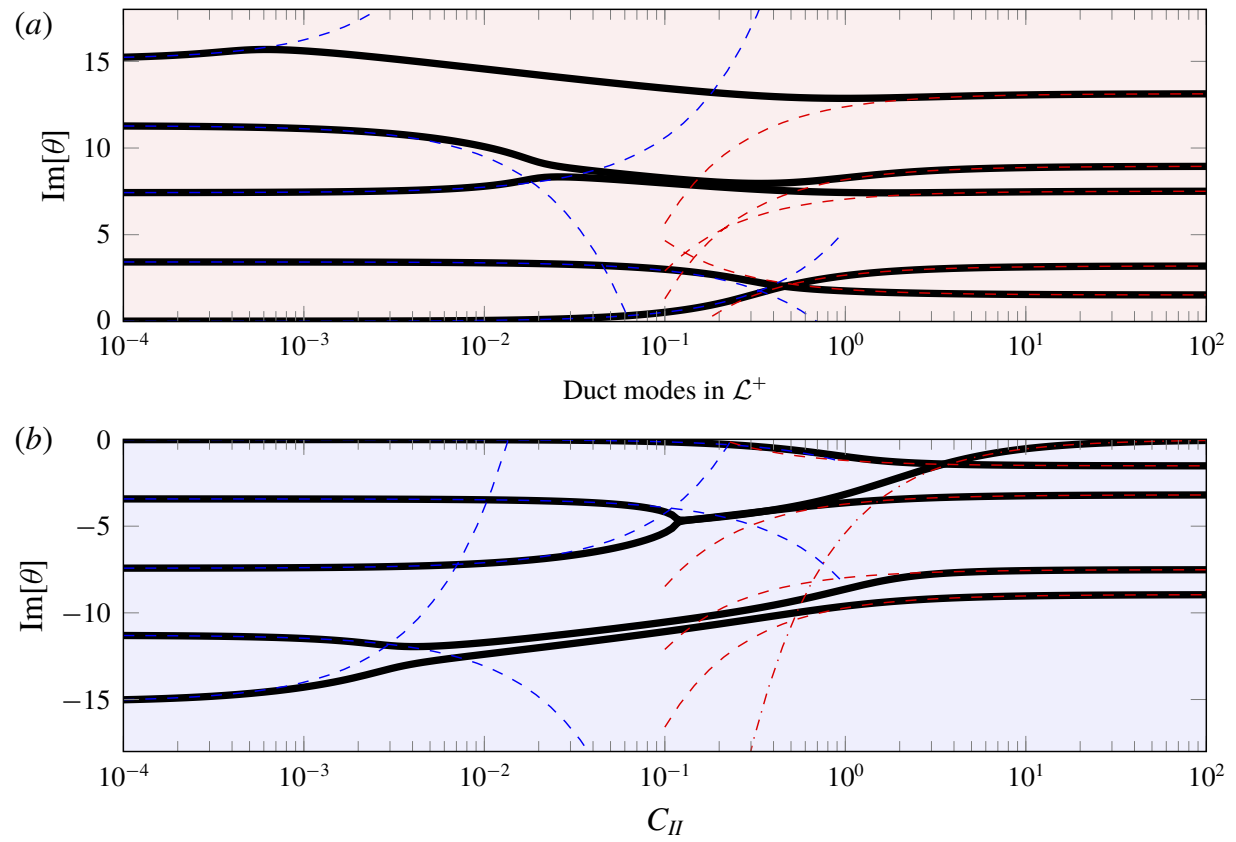

Duct modes in $\mathcal{L}^{-}$

FIGURE 8. The imaginary parts of the duct modes as a function of (real) porosity coefficient $C_{I I}$. The asymptotic approximations for small porosity coefficients (4.3) as illustrated by the dashed blue lines and the asymptotic approximations for large porosity coefficients $((4.4 a),(4.4 b))$ are denoted by the dashed red lines. The cascade parameters are defined in case $\mathrm{C}$ in table 2 with $\omega=5$ and $k_{x}=4$.

acoustic modes:

$$
\begin{gathered}
\theta_{0}=-\omega / \beta^{2}-\frac{1}{C_{I I}} \frac{\mathrm{i} \zeta\left(-\omega / \beta^{2}\right) \sin \left(s \zeta\left(-\omega / \beta^{2}\right)\right)}{\cos \left(s \zeta\left(-\omega / \beta^{2}\right)\right)-\cos \left(\sigma^{\prime}-d \omega / \beta^{2}\right)}+o\left(C_{I I}^{-1}\right), \\
\theta_{n}^{ \pm}=\lambda_{n}^{ \pm} \mp \frac{1}{C_{I I}} \frac{\mathrm{i}\left(\zeta_{n}^{ \pm}\right)^{2}}{\Delta\left(\lambda_{n}^{ \pm}+\omega / \beta^{2}\right) \sqrt{\omega^{2} w^{2}-f_{n}^{2}}}+o\left(C_{I I}^{-1}\right), \quad n \neq 0 .
\end{gathered}
$$

In figure 8 we illustrate the imaginary part of the duct modes as a function of porosity coefficient, along with our asymptotic approximations. These approximations are particularly accurate for the duct modes that are initially close to the real axis, and the accuracy deteriorates for modes with large imaginary part. The duct modes undergo rapid changes as $C_{I I}$ is increased to 1, which is suggests that large aeroacoustic gains can be made for modest porosity coefficients. This assertion is explored further in the following sections.

Further insight may be gained by examining the duct modes as a function of frequency for fixed porosity values. Figure 9 illustrates the duct modes for four porosity coefficients at a range of frequencies. For each porosity value, the imaginary part of the mode decreases as the frequency is increased. However, in contrast for case 0 blades, the imaginary part never vanishes for non-zero porosity values. In some cases (in $\mathcal{L}^{+}$), the imaginary part of the duct modes undergoes a slight increase before decreasing towards the real axis. The role of porosity is particularly important 

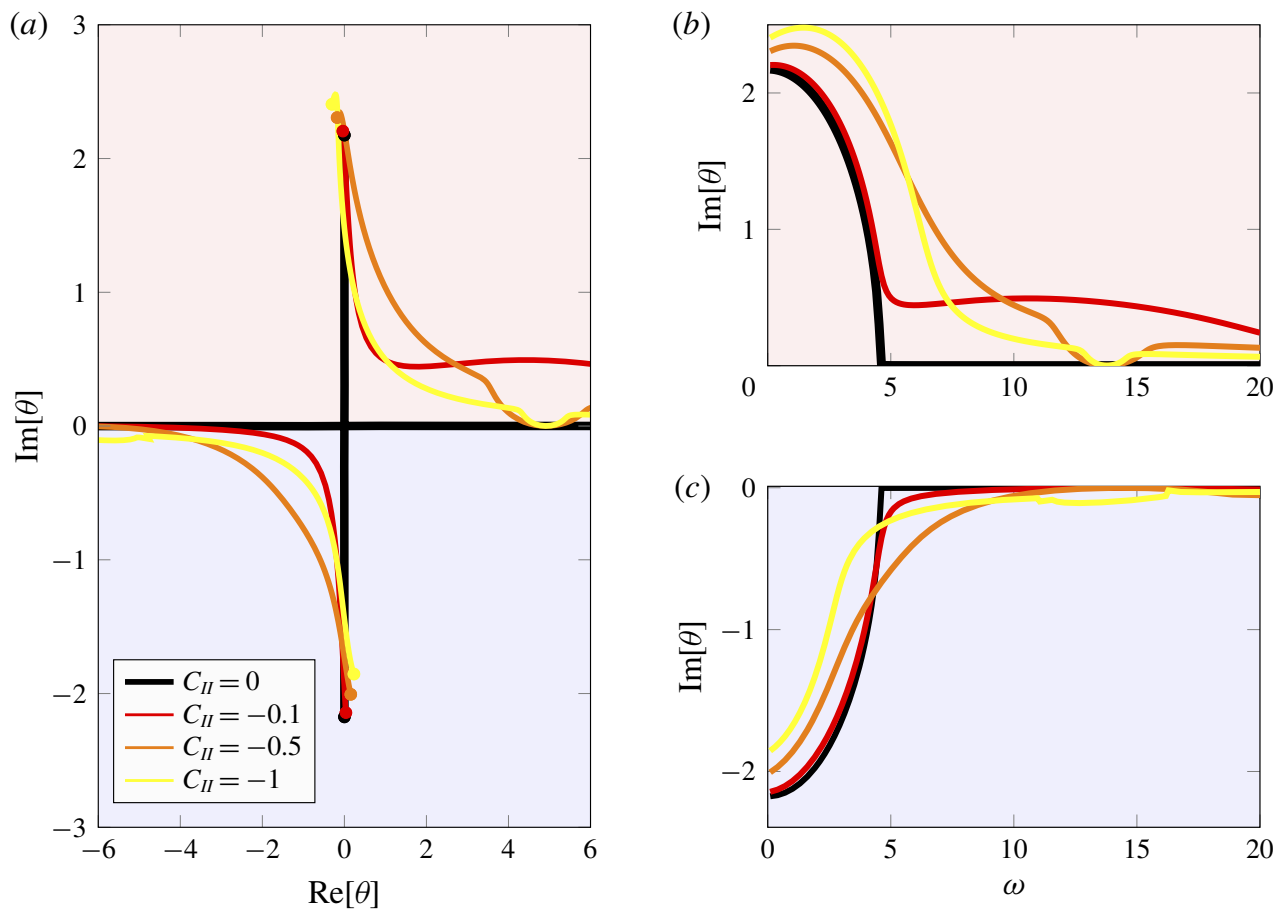

FIGURE 9. The trajectories of a pair of duct modes as the frequency is increased for a range of porosity constants. The duct modes for $\omega=0$ are denoted by $\bullet$. The complex trajectories are plotted in $(a)$, and the imaginary part of the modes in the upper and lower half planes are plotted in $(b, c)$ respectively. The colours correspond to different porosity parameters with black representing rigid and impermeable blades. The upper half-plane $\mathcal{L}^{+}$ is shaded in red and the lower half-plane $\mathcal{L}^{-}$is shaded in blue. The relevant parameters correspond to case $\mathrm{D}$ in table 2 with $k_{x}=2$.

for small to moderate frequencies. As the frequency is increased, the difference between the modes reduces.

We now consider the effect of porosity on sound generation and sound transmission.

\subsection{Sound generation}

Sound generation is caused when a pressure-free gust interacts with the blade row, resulting in the production of pressure waves. The incident perturbation can also be described as a 'vorticity wave' and is convected with the background flow so that $k_{x}=\omega$. In order to enable comparison against prior works, we consider cases analysed by Glegg (1999) and Posson et al. (2010) as defined in table 2. We consider cases where $C_{I I}<0$ so that the imaginary part of the Rayleigh conductivity is negative and the pores dissipate energy.

\subsubsection{Unsteady lift}

During the solution to the Wiener-Hopf problem associated with the scattering by a blade row with complex boundaries, we observed that the major difference with the rigid, impermeable case is that the duct modes are modified by the boundary conditions. Consequently, we expect complex boundary conditions to have 


$\begin{array}{lccccc} & \begin{array}{c}\text { Gap-to-chord } \\ \text { ratio }\end{array} & \begin{array}{c}\text { Stagger angle } \\ \chi / 2\end{array} & \begin{array}{ccc}\text { Mach number } \\ M_{0}\end{array} & \begin{array}{c}\text { Reduced } \\ \text { frequency }\end{array} & \begin{array}{c}\text { Inter-blade } \\ \text { phase angle }\end{array} \\ \text { Reference } & 0.6 & 40^{\circ} & 0.3 & 0-40 & \sigma \\ \text { Glegg (1999) } & 0.5 & 0^{\circ} & 0.5 & 5 \pi / 4 & 3 \pi / 4 \\ \text { Case A } & 0.5 & 0^{\circ} & 0.5 & 13 \pi / 4 & 13 \pi / 2 \\ \text { Case B } & 0.5 & 30^{\circ} & 0.3 & 5,10 & 2 \pi / 3 \\ \text { Case C } & 0.5 & 10^{\circ} & 0.4 & 0-20 & 4 \pi / 3 \\ \text { Case D } & 0.8 & 30^{\circ} & 0.1 & 100 & \pi / 4 \\ \text { Case E } & 0.8 & & \end{array}$

TABLE 2. Summary of parameters used in results section. All other parameters are taken to be zero, unless otherwise stated.

a significant effect on the unsteady loading of the blades. In this section we test that hypothesis.

The analytic expression for $D$ (3.11) enables the swift calculation of the unsteady loading on the blades. The unsteady loading is defined as the integral of the unsteady pressure over the blade surface

$$
C_{L}=\frac{1}{2 \pi w_{0}} \int_{0}^{2} \Delta_{0}[p](x) \mathrm{d} x .
$$

Integration by parts and application of the boundary conditions (3.10a) and (3.10c) yields the identity

$$
\begin{aligned}
D\left(-\omega M_{0}^{2} / \beta^{2}\right) & =\frac{1}{2 \pi} \int_{-\infty}^{\infty} \Delta_{0}[\phi](x) \mathrm{e}^{-\mathrm{i} \omega\left(M_{0} / \beta\right)^{2} x} \mathrm{~d} x \\
& =-\frac{1}{\mathrm{i} \omega 2 \pi} \int_{0}^{2} \frac{\partial}{\partial x}\left(\Delta_{0}[\phi](x) \mathrm{e}^{-\mathrm{i}\left(\omega / \beta^{2}\right) x}\right) \mathrm{e}^{\mathrm{i} \omega x} \mathrm{~d} x
\end{aligned}
$$

Consequently, the normalised unsteady lift (4.5) may be written as

$$
C_{L}=\frac{\mathrm{i} \omega}{w_{0}} D\left(-\omega M^{2} / \beta^{2}\right)
$$

The modified boundary conditions have a strong effect on the unsteady loading, as illustrated in figure 10. The unsteady loading for a case 0 cascade is compared against the loading for a range of porosity parameters, which correspond to the $C_{I I}$ values. The results indicate that the effect of the modified boundary conditions is to shift the locations of the duct modes, as indicated by the shifts in the local maximum around $\omega \approx 12$, which has previously been identified with the cut-on frequency of the duct mode (Glegg 1999). As $C_{I I}$ decreases, the pressure jump across the blade must decrease in accordance with the Rayleigh conductivity (2.15), thus ensuring that the seepage velocity through the blade is proportional to the pressure jump across the blade. This is observed in figure 10, where the effects of increasing the porosity result in an almost uniform reduction in the unsteady lift.

The impact of porosity on unsteady lift appears most pronounced when the frequency is low, which is fortuitous since the homogenised boundary condition (2.15) is most valid in the low-frequency regime. In particular, the range $0<\omega<7.62$ 


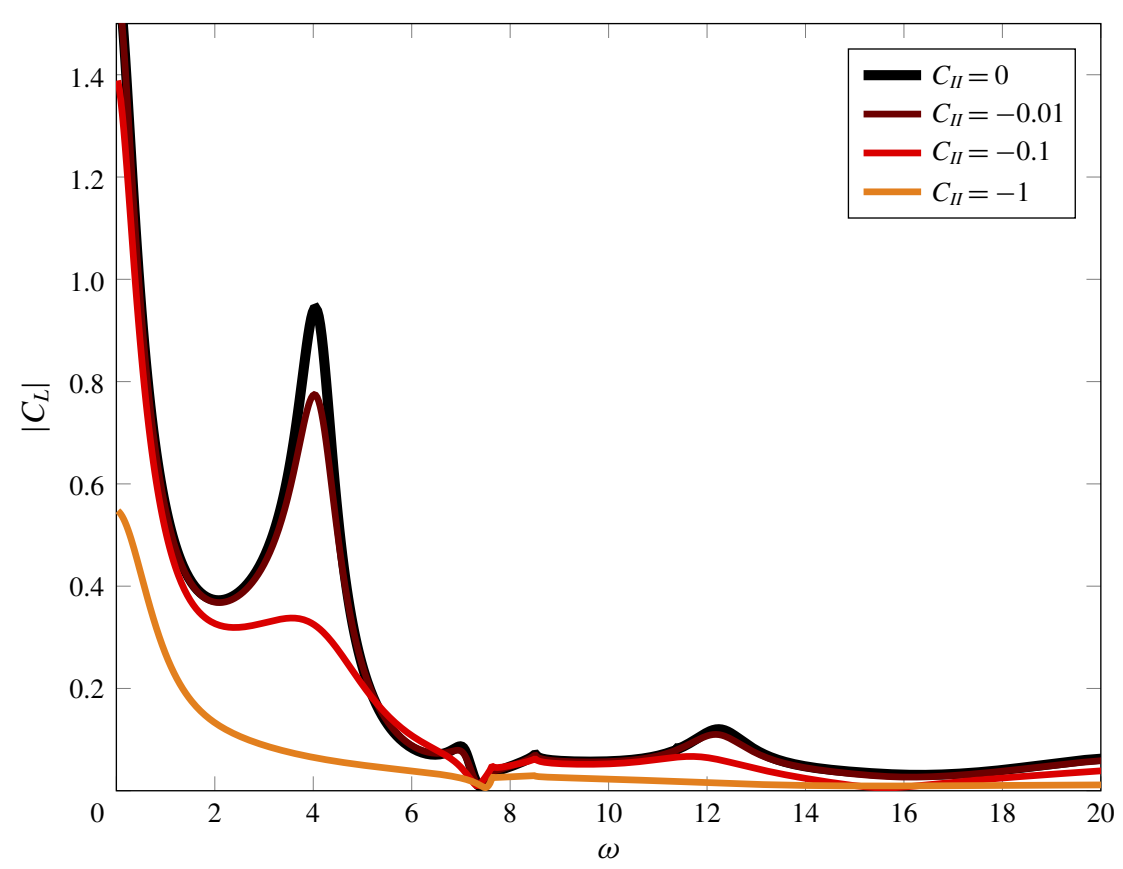

FIGURE 10. Unsteady lift for a range of frequencies and porosities. The aerodynamic and aeroacoustic parameters are defined in table 2 and correspond to those in figure 3 of Glegg (1999). The colours correspond to different porosity parameters with black representing rigid and impermeable blades.

shows the largest reduction in lift, and the peak around $\omega \approx 4$ is greatly attenuated. After the acoustic mode is cut-on at $\omega \approx 7.62$, the reductions in lift are generally smaller, but this is possibly because the lift on the case 0 cascade is also reduced; as a percentage, the reductions still appear significant.

\subsubsection{Sound power output}

Analytic expressions for the sound power output are available by a similar method to Glegg (1999). The modal upstream or downstream sound power output for the $m$ th mode is given by

$$
W_{ \pm}(m)=\frac{\omega \pi^{2}}{\Delta} \operatorname{Re}\left[\frac{\left|\zeta_{m}^{ \pm} D\left(\lambda_{m}^{ \pm}\right)\right|^{2}}{\sqrt{\omega^{2} w^{2}-f_{m}^{2}}}\right] .
$$

As noted multiple times in this paper, modifications to the surface boundary conditions do not affect the acoustic modes $\lambda_{m}^{ \pm}$. Consequently, the cut-on frequencies of these upstream and downstream modes are unaffected by porosity, for example. This is observed in figure 11(a), where the downstream sound power output for the first and second modes are illustrated for a large frequency range. Clearly the modes are cuton at the same frequency, but the magnitude of the sound power output is strongly affected by porosity. For small porosities $\left(C_{I I}=-0.01,-0.1\right)$ there is little impact on the sound power output of the first mode until the channel modes become cut-on at $\omega \approx 12$. Following this cut-on frequency, we observe a large decrease in the sound 

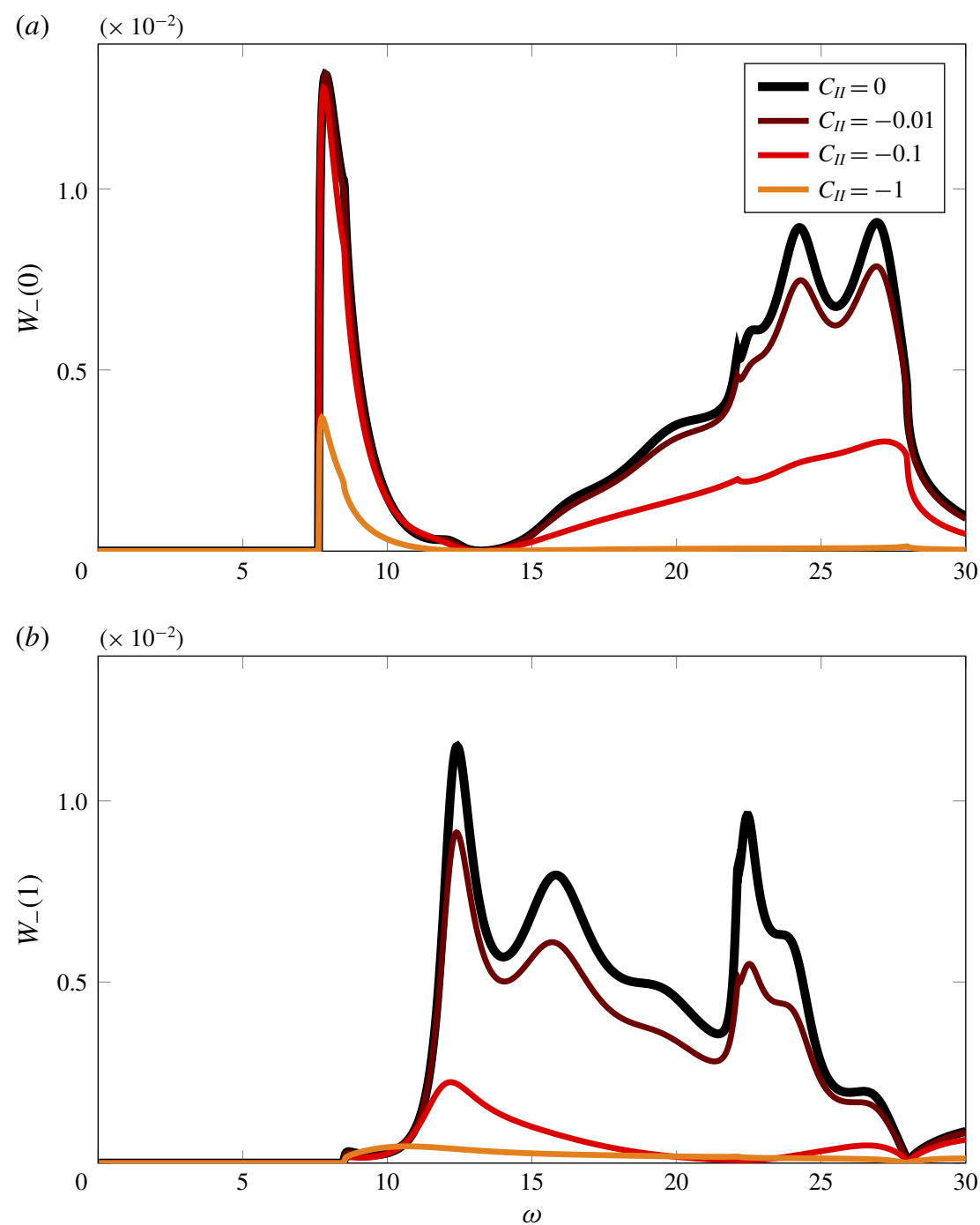

FIGURE 11. Modal downstream sound power output for a cascade of porous blades at a range of frequencies for $(a)$ the first mode $(m=0)$ and $(b)$ the second mode $(m=1)$. The aerodynamic and aeroacoustic parameters are defined in table 2 and correspond to those in figure 9 of Glegg (1999). The colours correspond to different porosity parameters with black representing rigid and impermeable blades.

power for all modes. Similarly to the unsteady lift, this reduction in sound power output can be attributed to the reduction in the pressure jump across the blade caused by porosity, thereby reducing the scattered sound in the upstream and downstream regions.

We define the sound power level as

$$
L_{W_{ \pm}}(m)=10 \log _{10}\left(\frac{W_{ \pm}(m)}{W_{r \pm}(m)}\right) \mathrm{dB},
$$



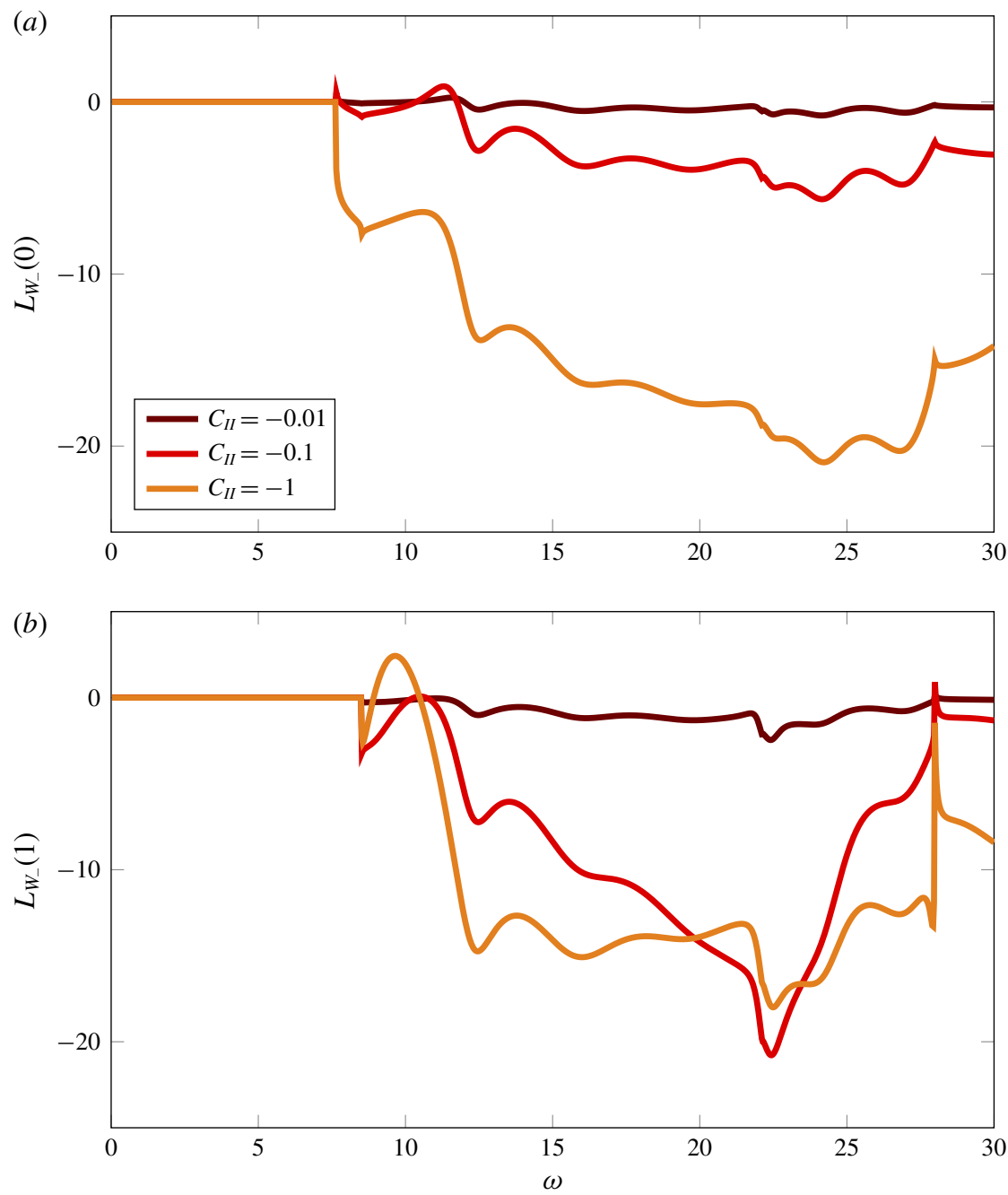

FIGURE 12. Modal downstream sound power levels for a cascade of porous blades at a range of frequencies for $(a)$ the first mode $(m=0)$ and $(b)$ the second mode $(m=1)$. The aerodynamic and aeroacoustic parameters are defined in table 2 and correspond to those in figure 9 of Glegg (1999). The colours correspond to different porosity parameters.

where we take the reference sound power $W_{r \pm}$ to be the sound power for a case 0 cascade. The reduction in sound power level is illustrated in figure 12 for a range of porosity parameters. We observe that even a modest porosity of $C_{I I}=-0.1$ is capable of large sound power level reductions of $5 \mathrm{~dB}$ for the first mode and $20 \mathrm{~dB}$ for the second mode.

\subsection{Sound transmission}

We now use our model to investigate sound transmission through a cascade of flat plates with complex boundaries. Sound transmission occurs when a sound wave interacts with a cascade of blades, and is reflected and transmitted through the blade row. In order to explore the effects of modified boundary conditions on the 
transmission and reflection we write the incident acoustic field (2.6) in the form

$$
\phi_{i}=\mathrm{e}^{\mathrm{i} \zeta_{0}^{-} y} \mathrm{e}^{-\mathrm{i} \lambda_{0}^{-} x}, \quad x \leqslant y d / s
$$

where the mode is assumed cut-on, so that $\lambda_{0}$ is real. Comparison with the solution in $\S 3.1$ shows that the reflected acoustic potential takes the form

$$
\phi_{r}=\sum_{m=-\infty}^{\infty} R_{m} \mathrm{e}^{\mathrm{i} \zeta_{m}^{+} y} \mathrm{e}^{-\mathrm{i} \lambda_{m}^{+} x}, \quad x \leqslant y d / s,
$$

and the transmitted acoustic potential takes the form

$$
\phi_{t}=\sum_{m=-\infty}^{\infty} T_{m} \mathrm{e}^{-\mathrm{i} \zeta_{m}^{-} y} \mathrm{e}^{-\mathrm{i} \lambda_{m}^{-}(x-2)}, \quad x \geqslant 2+y d / s,
$$

where $R_{m}$ and $T_{m}$ are the reflection and transmission coefficients respectively. Note that the hydrodynamic mode $\left(\omega / \beta^{2}\right)$ corresponding to the jump in acoustic potential across the wake is not included in this analysis since it does not contribute to the pressure field.

By comparison with (3.12) and (3.13) we obtain the expressions for the transmission and reflection coefficients as

$$
R_{m}=+\frac{\pi \zeta_{m}^{+} D^{(1,3)}\left(\lambda_{m}^{+}\right)}{\Delta \sqrt{\omega^{2} w^{2}-f_{m}^{2}}}, \quad T_{m}=-\frac{\pi \zeta_{m}^{-} D^{(2,4)}\left(\lambda_{m}^{-}\right)}{\Delta \sqrt{\omega^{2} w^{2}-f_{m}^{2}}} \mathrm{e}^{-2 \mathrm{i} \lambda_{m}^{-}}+\delta_{0, m} .
$$

We now consider a practical example. Suppose that the blade is perforated with holes of radius $R^{*}=0.02 b^{*}$. We consider perturbations such that the pore-scale reduced frequency is $\omega_{P}=\omega^{*} R^{*} / U^{*}=2$, which corresponds to a reduced frequency of $\omega=100$ in our notation. Howe et al. (1996) calculated the components of the Rayleigh conductivity in this case to be $\Gamma_{R}=1.252$ and $\Delta_{R}=0.705$, so that $K_{R}=0.0250-0.0141 \mathrm{i}$. The colormaps in figure 13(ai-ci) show the pressure field for three different open area fractions. The case 0 (rigid and impermeable) cascade shows significant diffraction and reflection of the incident wave, as indicated by the relatively large reflection and transmission coefficients in figure 13(aii). Increasing the open area fraction to the modest value of $\alpha_{H}=0.01$ slightly decreases the strength of the reflected and transmitted waves, as evidenced in figure 13(bii). Increasing the open area fraction further to $\alpha_{H}=0.05$ (figure 13cii) almost completely annihilates the backscattering. The effect is similar in the downstream field, though the open area fraction appears to have a slightly less significant effect, at least for the parameters we consider here. This asymmetry can be attributed to the Kutta condition. In contrast to the leading edge where the pressure is singular, at the trailing edge we require that the pressure jump vanishes. Accordingly, increasing the open area fraction cannot reduce the pressure jump by the same magnitude at the trailing edge as at the leading edge.

Since the Rayleigh conductivity has negative imaginary part, the incident perturbation transfers energy to the mean flow by means of the viscous action at the pore scale. Howe et al. (1996) provide a more detailed treatment of this phenomenon, and showed the perturbations can actually be amplified in certain frequency bands when the imaginary part of the Rayleigh conductivity is positive. This suggests that acoustic energy can only be absorbed by porous blades in certain frequency regimes, which is in accordance with numerical experiments we performed for the cascade. 

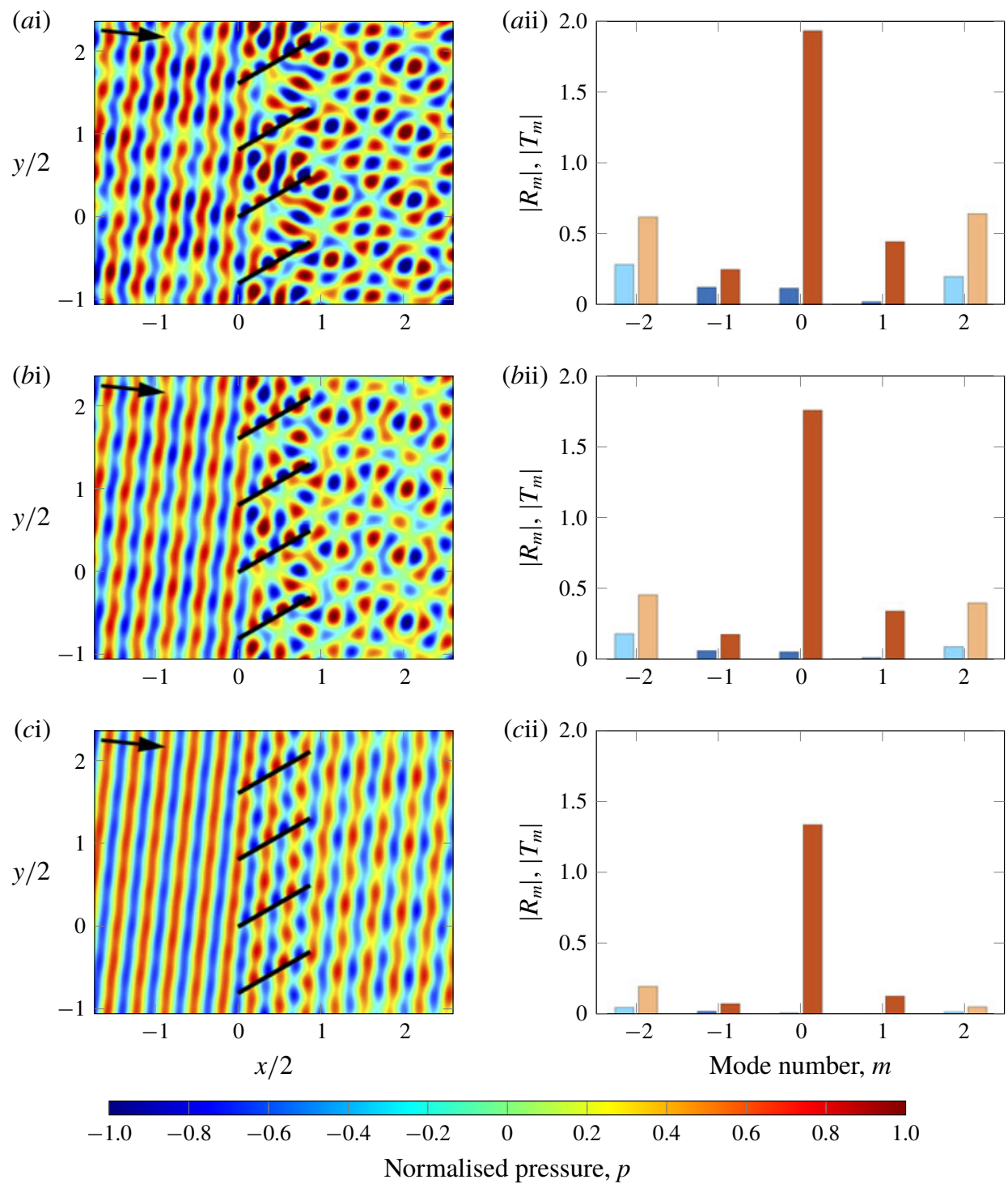

FIGURE 13. (ai-ci) The total (incident and scattered), normalised pressure field. (aii-cii) The amplitudes of the normalised reflection (blue) and transmission (orange) coefficients. The open area fraction values are $\alpha_{H}=0,0.01$ and 0.05 respectively. The darker bars indicate modes that are cut-on whereas the lighter bars are cut-off. The parameters in this case are defined in case $\mathrm{E}$ of table 2. The arrow indicates the direction of the incident wave.

\section{Conclusions}

We have derived an analytic solution for the scattering of an unsteady perturbation incident on a rectilinear cascade of flat blades with complex boundaries. The analytic nature of the solution means that it is extremely rapid to compute, and offers physical insight into the role played by different boundary conditions. In contrast with previous studies that focussed on the effects of rigid and impermeable blades (Glegg 1999; 
Posson et al. 2010), the formulation of the present research allows a range of boundary conditions to be studied with minimal effort, such as porosity, compliance and flow impedance. In terms of the spectral plane, the effect of modifying these boundary conditions is to change the locations of the zeros of the Wiener-Hopf kernel, whilst the poles are unchanged. Accordingly, the modal structure of the far-field scattered pressure is invariant under modifications to the flat blades' boundary conditions. Conversely, the modal structure of the near-field region undergoes large deformations since the zeros of the kernel correspond to the duct modes of the inter-blade region. This has a strong effect on the surface pressure fluctuations and unsteady loading, which has implications for the far-field sound.

We have focussed on understanding the role of blade porosity. The results show that substantial reductions in both the unsteady lift and sound power output are possible for even modest values of porosity. At low frequencies we observe a significant change in the unsteady loading and a moderate effect on the sound power output. Conversely, at high frequencies we observe a significant effect on the sound power output and a small effect on the unsteady loading. Furthermore, the amplitudes of the reflection and transmission coefficients rapidly decrease as the blade porosity is increased. We attribute these considerable reductions to several physical mechanisms associated with porosity. Increasing the blade porosity promotes the communication between adjacent channels, which prevents large build-ups of pressure inside each channel. Moreover, the flow seepage afforded by porous channels permits the dissipation of energy through the blades, thus reducing unsteady pressure fluctuations on the blade. This reduction in pressure fluctuations corresponds to reductions in the scattered pressure and far-field sound. This study shows that modified boundary conditions can have a large impact on the generation and transmission of sound in cascades, and our analytic solution offers a useful design tool that models both aeroacoustic and aeroelastic effects. Future work will focus on understanding how surface treatments can be used to change the resonant frequency of a cascade to avoid lock-in and the associated structural damage.

\section{Declaration of interests}

The authors report no conflict of interest.

\section{Appendix A. Wiener-Hopf solution}

We now solve the integral equation (3.8) subject to the boundary conditions of no discontinuities upstream (2.7), a modified no-flux condition (2.17) and no pressure jump across the wake (2.20). In a similar way to Glegg (1999), we split this problem into four coupled problems that are amenable to the Wiener-Hopf method.

We write

$$
\Delta_{0}[\phi](x)=\Delta_{0}\left[\phi^{(1)}\right](x)+\Delta_{0}\left[\phi^{(2)}\right](x)+\Delta_{0}\left[\phi^{(3)}\right](x)+\Delta_{0}\left[\phi^{(4)}\right](x),
$$

and its Fourier transform

$$
D(\gamma)=D^{(1)}(\gamma)+D^{(2)}(\gamma)+D^{(3)}(\gamma)+D^{(4)}(\gamma),
$$

where each $\Delta_{0}\left[\phi^{(n)}\right]$ and $D^{(n)}$ satisfy a semi-infinite integral equation of the form

$$
f^{(n)}(x)=4 \pi \int_{-\infty}^{\infty} D^{(n)}(\gamma) j(\gamma) \mathrm{e}^{-\mathrm{i} \gamma x} \mathrm{~d} \gamma,
$$


for $n=1,2,3,4$. The corresponding boundary conditions are

$$
\begin{gathered}
f^{(1)}(x)=\mu_{0} \Delta_{0}\left[\phi^{(1)}\right](x)+\mu_{1} \Delta_{0}\left[\phi_{x}^{(1)}\right](x)+\mu_{2} \Delta_{0}\left[\phi_{x, x}^{(1)}\right](x) \\
-2 w_{0} \exp \left[\mathrm{i}\left(k_{x} / \beta^{2}\right) x\right], \quad x>0, \\
f^{(2)}(x)=\mu_{0} \Delta_{0}\left[\phi^{(2)}\right](x)+\mu_{1} \Delta_{0}\left[\phi_{x}^{(2)}\right](x)+\mu_{2} \Delta_{0}\left[\phi_{x, x}^{(2)}\right](x), \quad x<2, \\
f^{(3)}(x)=\mu_{0} \Delta_{0}\left[\phi^{(3)}\right](x)+\mu_{1} \Delta_{0}\left[\phi_{x}^{(3)}\right](x)+\mu_{2} \Delta_{0}\left[\phi_{x, x}^{(3)}\right](x), \quad x>0, \\
f^{(4)}(x)=\mu_{0} \Delta_{0}\left[\phi^{(4)}\right](x)+\mu_{1} \Delta_{0}\left[\phi_{x}^{(4)}\right](x)+\mu_{2} \Delta_{0}\left[\phi_{x, x}^{(4)}\right](x), \quad x<2,
\end{gathered}
$$

and

$$
\begin{gathered}
\Delta_{0}\left[\phi^{(1)}\right](x)=0, \quad x<0, \\
\Delta_{0}\left[\phi^{(1)}\right](x)+\Delta_{0}\left[\phi^{(2)}\right](x)=2 \pi \mathrm{i} P^{(2)} \mathrm{e}^{\mathrm{i}\left(\omega / \beta^{2}\right) x}, \quad x>2, \\
\Delta_{0}\left[\phi^{(2)}\right](x)+\Delta_{0}\left[\phi^{(3)}\right](x)+\Delta_{0}\left[\phi^{(4)}\right](x)=0, \quad x<0, \\
\Delta_{0}\left[\phi^{(3)}\right](x)+\Delta_{0}\left[\phi^{(4)}\right](x)=2 \pi \mathrm{i} P^{(4)} \mathrm{e}^{\mathrm{i}\left(\omega / \beta^{2}\right) x}, \quad x>2,
\end{gathered}
$$

where $P^{(2)}$ and $P^{(4)}$ are two constants of integration that will be specified to enforce the Kutta condition. Summing the four above conditions results in the original boundary conditions and, consequently, we may apply the Wiener-Hopf method to each semi-infinite integral equation and sum the resulting contributions to obtain a solution to the original equations.

\section{A.1. Solution to first Wiener-Hopf equation $-D^{(1)}$}

In this section, we solve the integral equation (A 3) for $n=1$

$$
f^{(1)}(x)=4 \pi \int_{-\infty}^{\infty} D^{(1)}(\gamma) j(\gamma) \mathrm{e}^{-\mathrm{i} \gamma x} \mathrm{~d} \gamma,
$$

subject to (A 4a) and (A 4b). Taking a Fourier transform of (A 8) in $x$ gives

$$
F_{-}^{(1)}(\gamma)+F_{+}^{(1)}(\gamma)=4 \pi D_{+}^{(1)}(\gamma) j(\gamma)
$$

where

$$
\begin{gathered}
F_{-}^{(1)}(\gamma)=\frac{1}{2 \pi} \int_{-\infty}^{0} f^{(1)}(x) \mathrm{e}^{\mathrm{i} \gamma x} \mathrm{~d} x, \quad F_{+}^{(1)}(\gamma)=\frac{1}{2 \pi} \int_{0}^{\infty} f^{(1)}(x) \mathrm{e}^{\mathrm{i} \gamma x} \mathrm{~d} x \\
D_{+}^{(1)}(\gamma)=\int_{0}^{\infty} \Delta_{0}\left[\phi^{(1)}\right](x) \mathrm{e}^{\mathrm{i} \gamma x} \mathrm{~d} x
\end{gathered}
$$


The subscripts \pm here indicate that the functions are analytic in the upper or lower half planes, denoted by $\mathcal{L}^{ \pm}$. We may employ (A $4 a$ ) to obtain

$$
F_{+}^{(1)}(\gamma)=\frac{w_{0}}{\pi \mathrm{i}\left(\gamma+k_{x} / \beta^{2}\right)}+\left(\mu_{0}-\mathrm{i} \mu_{1} \gamma-\mu_{2} \gamma^{2}\right) D^{(1)}(\gamma) \text {. }
$$

Consequently, the Wiener-Hopf equation (A 9) may be expressed as

$$
F_{-}^{(1)}(\gamma)+\frac{w_{0}}{\pi \mathrm{i}\left(\gamma+k_{x} / \beta^{2}\right)}=4 \pi D_{+}^{(1)}(\gamma) K(\gamma),
$$

where

$$
K(\gamma)=j(\gamma)-\frac{1}{4 \pi}\left(\mu_{0}-\mathrm{i} \mu_{1} \gamma-\mu_{2} \gamma^{2}\right)
$$

The multiplicative splitting $K=K_{+} K_{-}$is performed in appendix B. This splitting enables us to write

$$
\frac{F_{-}^{(1)}(\gamma)}{K_{-}(\gamma)}+\frac{w_{0}}{\pi \mathrm{i}\left(\gamma+k_{x} / \beta^{2}\right) K_{-}(\gamma)}=4 \pi D_{+}^{(1)}(\gamma) K_{+}(\gamma)
$$

We now additively factorise the left side of (A 13). We apply pole removal (Noble 1958) to obtain the additive splitting

$$
\begin{aligned}
\frac{1}{\left(\gamma+k_{x} / \beta^{2}\right) K_{-}(\gamma)}= & \frac{1}{\underbrace{\left(\gamma+k_{x} / \beta^{2}\right) K_{-}\left(-k_{x} / \beta^{2}\right)}_{+}} \\
& +\underbrace{\frac{1}{\left(\gamma+k_{x} / \beta^{2}\right) K_{-}(\gamma)}-\frac{1}{\left(\gamma+k_{x} / \beta^{2}\right) K_{-}\left(-k_{x} / \beta^{2}\right)}}_{-},
\end{aligned}
$$

where the underbrace \pm denotes that the function is analytic in $\mathcal{L}^{ \pm}$respectively. Therefore, (A 11) becomes

$$
\begin{gathered}
\frac{F_{-}^{(1)}(\gamma)}{K_{-}(\gamma)}+\frac{w_{0}}{\pi \mathrm{i}\left(\gamma+k_{x} / \beta^{2}\right) K_{-}(\gamma)}-\frac{w_{0}}{\pi \mathrm{i}\left(\gamma+k_{x} / \beta^{2}\right) K_{-}\left(-k_{x} / \beta^{2}\right)} \\
=4 \pi D_{+}^{(1)}(\gamma) K_{+}(\gamma)-\frac{w_{0}}{\pi \mathrm{i}\left(\gamma+k_{x} / \beta^{2}\right) K_{-}\left(-k_{x} / \beta^{2}\right)} .
\end{gathered}
$$

We may now apply the standard Wiener-Hopf argument: since the left and right sides of (A 14) are analytic in $\mathcal{L}^{\mp}$ respectively, and they agree on a strip, each side defines the analytic continuation of the other. Therefore, equation (A 14) defines an entire function, $E_{1}(\gamma)$. As $|\gamma| \rightarrow \infty$ in $\mathcal{L}^{-}$, the left side of (A 14) decays. This can be seen by considering the growth of $K_{-}$, which is explicated in appendix B. Similarly, as $|\gamma| \rightarrow \infty$ in $\mathcal{L}^{+}$, the right side of (A 14) vanishes. Therefore, $E_{1}(\gamma)$ is bounded in the entire plane and must be constant according to Liouville's theorem. Moreover, since $E_{1}(\gamma)$ decays, this constant must be zero. Finally, we rearrange the right side of (A 14) to obtain the solution to the first Wiener-Hopf problem as

$$
D^{(1)}(\gamma)=\frac{w_{0}}{(2 \pi)^{2} \mathrm{i}\left(\gamma+k_{x} / \beta^{2}\right) K_{-}\left(-k_{x} / \beta^{2}\right) K_{+}(\gamma)} .
$$


A.2. Solution to second Wiener-Hopf equation $-D^{(2)}$

In this section we solve the integral equation (A 3) for $n=2$,

$$
f^{(2)}(x)=4 \pi \int_{-\infty}^{\infty} D^{(2)}(\gamma) j(\gamma) \mathrm{e}^{-\mathrm{i} \gamma x} \mathrm{~d} \gamma
$$

subject to (A 5a) and (A 5b). Taking a Fourier transform of (A 16a) and applying (A $5 a$ ) yields

$$
\left(\mu_{0}-\mathrm{i} \mu_{1} \gamma-\mu_{2} \gamma^{2}\right) D^{(1)}(\gamma)+F_{+}^{(2)}(\gamma)=4 \pi\left(D_{-}^{(2)}(\gamma)+D_{+}^{(2)}(\gamma)\right) j(\gamma),
$$

where

$$
\begin{gathered}
F_{+}^{(2)}(\gamma)=\frac{1}{2 \pi} \int_{2}^{\infty} f^{(2)}(x) \mathrm{e}^{\mathrm{i} \gamma x} \mathrm{~d} x=\frac{\mathrm{e}^{2 \mathrm{i} \gamma}}{2 \pi} \int_{0}^{\infty} f^{(2)}(x+2) \mathrm{e}^{\mathrm{i} x \gamma} \mathrm{d} x=\mathrm{e}^{2 \mathrm{i} \gamma} F_{+}^{*(2)}(\gamma), \quad(\mathrm{A} 18 a) \\
D_{+}^{(2)}(\gamma)=\frac{1}{2 \pi} \int_{2}^{\infty} \Delta_{0}\left[\phi^{(2)}\right](x) \mathrm{e}^{\mathrm{i} \gamma x} \mathrm{~d} x=\frac{\mathrm{e}^{2 \mathrm{i} \gamma}}{2 \pi} \int_{0}^{\infty} \Delta_{0}\left[\phi^{(2)}\right](x+2) \mathrm{e}^{\mathrm{i} x \gamma} \mathrm{d} x=\mathrm{e}^{2 \mathrm{i} \gamma} D_{+}^{*(2)}(\gamma), \\
D_{-}^{(2)}(\gamma)=\frac{1}{2 \pi} \int_{-\infty}^{2} \Delta_{0}\left[\phi^{(2)}\right](x) \mathrm{e}^{\mathrm{i} \gamma x} \mathrm{~d} x=\frac{\mathrm{e}^{2 \mathrm{i} \gamma}}{2 \pi} \int_{-\infty}^{0} \Delta_{0}\left[\phi^{(2)}\right](x+2) \mathrm{e}^{\mathrm{i} x \gamma} \mathrm{d} x=\mathrm{e}^{2 \mathrm{i} \gamma} D_{-}^{*(2)}(\gamma) .
\end{gathered}
$$

Factoring out the $\mathrm{e}^{2 \mathrm{i} \gamma}$ dependence and employing (A 12) transforms the Wiener-Hopf equation (A 19) to

$$
F_{+}^{*(2)}(\gamma)=4 \pi\left(D_{-}^{*(2)}(\gamma)+D_{+}^{*(2)}(\gamma)\right) K(\gamma),
$$

and we may use the multiplicative splitting of $K$ to write

$$
\frac{F_{+}^{*(2)}(\gamma)}{K_{+}(\gamma)}=4 \pi\left(D_{-}^{*(2)}(\gamma)+D_{+}^{*(2)}(\gamma)\right) K_{-}(\gamma) \text {. }
$$

We may use the downstream boundary condition for this problem (A $5 b$ ) to write

$$
D_{+}^{*(2)}(\gamma)=-\frac{P^{*(2)}}{\gamma+\omega / \beta^{2}}-\frac{1}{2 \pi} \int_{0}^{\infty} \Delta_{0}\left[\phi^{(1)}\right](x+2) \mathrm{e}^{\mathrm{i} \gamma x} \mathrm{~d} x
$$

where $P^{*(2)}=P^{(2)} \mathrm{e}^{2 \mathrm{i} k_{x} / \beta^{2}}$. To calculate the remaining integral we use the inversion formula for the Fourier transform

$$
\Delta_{0}\left[\phi^{(1)}\right](x)=\int_{-\infty-\mathrm{i} \tau_{1}}^{\infty-\mathrm{i} \tau_{1}} D^{(1)}(\gamma) \mathrm{e}^{-\mathrm{i} \gamma x} \mathrm{~d} \gamma
$$

for some $\tau_{1}>0$. By substituting this representation into our desired integral, we obtain

$$
\frac{1}{2 \pi} \int_{0}^{\infty} \Delta_{0}\left[\phi^{(1)}\right](x+2) \mathrm{e}^{\mathrm{i} \gamma x} \mathrm{~d} x=\frac{1}{2 \pi} \int_{0}^{\infty} \int_{-\infty-\mathrm{i} \tau_{1}}^{\infty-\mathrm{i} \tau_{1}} D^{(1)}\left(\gamma_{1}\right) \mathrm{e}^{-\mathrm{i} \gamma_{1}(x+2)} \mathrm{d} \gamma_{1} \mathrm{e}^{\mathrm{i} \gamma x} \mathrm{~d} x .
$$

Rearranging the order of integration and computing the resulting $x$-integral results in

$$
\frac{1}{2 \pi} \int_{0}^{\infty} \Delta_{0}\left[\phi^{(1)}\right](x+2) \mathrm{e}^{\mathrm{i} \gamma x} \mathrm{~d} x=\frac{1}{2 \pi \mathrm{i}} \int_{-\infty-\mathrm{i} \tau_{1}}^{\infty-\mathrm{i} \tau_{1}} \frac{D^{(1)}\left(\gamma_{1}\right) \mathrm{e}^{-2 \mathrm{i} \gamma_{1}}}{\gamma_{1}-\gamma} \mathrm{d} \gamma_{1} .
$$


Since $d<2$, we may close the remaining integral in $\mathcal{L}^{-}$. There are no branches in the integrand so the integral consists of the residues of simple poles at $\gamma=-k_{x} / \beta^{2}$, $\theta_{n}^{-}$. Consequently, inserting (A 15) into the above integral yields

$$
\begin{aligned}
& \frac{1}{2 \pi \mathrm{i}} \int_{-\infty-\mathrm{i} \tau_{1}}^{\infty-\mathrm{i} \tau_{1}} \frac{1}{\left(\gamma+k_{x} / \beta^{2}\right) K_{-}\left(-k_{x} / \beta^{2}\right) K_{+}(\gamma)} \frac{\mathrm{e}^{-2 \mathrm{i} \gamma_{1}}}{\gamma_{1}-\gamma} \mathrm{d} \gamma_{1} \\
& \quad=\frac{\mathrm{e}^{2 \mathrm{i} k_{x} / \beta^{2}}}{\left(\gamma+k_{x} / \beta^{2}\right) K\left(-k_{x} / \beta^{2}\right)}+\sum_{n=0}^{\infty} \frac{\mathrm{e}^{-2 \mathrm{i} \theta_{n}^{-}}}{\left(\theta_{n}^{-}+k_{x} / \beta^{2}\right) K_{-}\left(-k_{x} / \beta^{2}\right) K_{+}^{\prime}\left(\theta_{n}^{-}\right)\left(\gamma-\theta_{n}^{-}\right)},
\end{aligned}
$$

where the derivatives of $K_{ \pm}$evaluated at the duct modes $\theta_{n}^{\mp}$ are given by

$$
\begin{aligned}
K_{ \pm}^{\prime}\left(\theta_{n}^{\mp}\right)= & \frac{K^{\prime}\left(\theta_{n}^{\mp}\right)}{K_{\mp}\left(\theta_{n}^{\mp}\right)}=\frac{1}{4 \pi K_{\mp}\left(\theta_{n}^{\mp}\right)}\left(-\frac{\left(\theta_{n}^{\mp} / \zeta\left(\theta_{n}^{\mp}\right)\right) \sin \left(s \zeta\left(\theta_{n}^{\mp}\right)\right)+s \theta_{n}^{\mp} \cos \left(s \zeta\left(\theta_{n}^{\mp}\right)\right)}{\cos \left(s \zeta\left(\theta_{n}^{\mp}\right)\right)-\cos \left(d \theta_{n}^{\mp}+\sigma^{\prime}\right)}\right. \\
& -\frac{\theta_{n}^{\mp} s \sin \left(s \zeta\left(\theta_{n}^{\mp}\right)\right)^{2}+d \zeta\left(\theta_{n}^{\mp}\right) \sin \left(d \theta_{n}^{\mp}+\sigma^{\prime}\right) \sin \left(s \zeta\left(\theta_{n}^{\mp}\right)\right)}{\left(\cos \left(s \zeta\left(\theta_{n}^{\mp}\right)\right)-\cos \left(d \theta_{n}^{\mp}+\sigma^{\prime}\right)\right)^{2}} \\
& \left.+\mathrm{i} \mu_{1}+2 \mu_{2} \theta_{n}^{\mp}\right) .
\end{aligned}
$$

Therefore, substitution of (A 22) into (A 21) yields

$$
\begin{aligned}
D_{+}^{*(2)}(\gamma)= & \frac{w_{0} \mathrm{e}^{2 \mathrm{i} k_{x} / \beta^{2}}}{(2 \pi)^{2} \mathrm{i}\left(\gamma+k_{x} / \beta^{2}\right) K\left(-k_{x} / \beta^{2}\right)}-\sum_{n=0}^{\infty} \frac{\mathcal{A}_{n} \mathrm{e}^{-2 \mathrm{i} \theta_{n}^{-}}}{\mathrm{i}\left(\theta_{n}^{-}+\omega / \beta^{2}\right)\left(\gamma-\theta_{n}^{-}\right)}, \\
& -\frac{P^{*(2)}}{\gamma+\omega / \beta^{2}},
\end{aligned}
$$

where

$$
\mathcal{A}_{n}=\frac{w_{0}\left(\theta_{n}^{-}+\omega / \beta^{2}\right)}{(2 \pi)^{2}\left(\theta_{n}^{-}+k_{x} / \beta^{2}\right) K_{-}\left(-k_{x} / \beta^{2}\right) K_{+}^{\prime}\left(\theta_{n}^{-}\right)} .
$$

We use the notation

$$
\tilde{K}_{-}\left(\gamma, \eta^{-}\right) \triangleq \frac{K_{-}(\gamma)}{\gamma-\eta^{-}},
$$

so that substitution of (A 23) into the Wiener-Hopf equation (A 17) yields

$$
\begin{aligned}
\frac{F_{+}^{*(2)}(\gamma)}{4 \pi K_{+}(\gamma)}= & K_{-}(\gamma) D_{-}^{*(2)}(\gamma)-P^{*(2)} \tilde{K}_{-}\left(\gamma,-\omega / \beta^{2}\right) \\
& +\frac{w_{0} \mathrm{e}^{2 \mathrm{i} k_{x} / \beta^{2}}}{(2 \pi)^{2} \mathrm{i}} \frac{\tilde{K}_{-}\left(\gamma,-k_{x} / \beta^{2}\right)}{K\left(-k_{x} / \beta^{2}\right)}-\sum_{n=0}^{\infty} \frac{\mathcal{A}_{n} \mathrm{e}^{-2 \mathrm{i} \theta_{n}^{-}}}{\mathrm{i}\left(\theta_{n}^{-}+\omega / \beta^{2}\right)} \tilde{K}_{-}\left(\gamma, \theta_{n}^{-}\right) .
\end{aligned}
$$

We note the additive splitting

$$
\tilde{K}_{-}\left(\gamma, \eta^{-}\right)=\left[\tilde{K}_{-}(\gamma, \eta)\right]_{+}+\left[\tilde{K}_{-}(\gamma, \eta)\right]_{-},
$$


where

$$
\left[\tilde{K}_{-}(\gamma, \eta)\right]_{+}=\frac{K_{-}\left(\eta^{-}\right)}{\gamma-\eta^{-}}, \quad\left[\tilde{K}_{-}(\gamma, \eta)\right]_{-}=\frac{K_{-}(\gamma)-K_{-}\left(\eta^{-}\right)}{\gamma-\eta^{-}} .
$$

Substituting these splittings into (A 24) yields

$$
\begin{aligned}
& \frac{F_{+}^{*(2)}(\gamma)}{4 \pi K_{+}(\gamma)}+P^{*(2)}\left[\tilde{K}_{-}\left(\gamma,-\omega / \beta^{2}\right)\right]_{+}-\frac{w_{0} \mathrm{e}^{2 \mathrm{i} k_{x} / \beta^{2}}}{(2 \pi)^{2} \mathrm{i}} \frac{\left[\tilde{K}_{-}\left(\gamma,-k_{x} / \beta^{2}\right)\right]_{+}}{K\left(-k_{x} / \beta^{2}\right)} \\
& \quad+\sum_{n=0}^{\infty} \frac{\mathcal{A}_{n} \mathrm{e}^{-2 \mathrm{i} \theta_{n}^{-}}}{\mathrm{i}\left(\theta_{n}^{-}+\omega / \beta^{2}\right)}\left[\tilde{K}_{-}\left(\gamma, \theta_{n}^{-}\right)\right]_{+}=K_{-}(\gamma) D_{-}^{*(2)}(\gamma)-P^{*(2)}\left[\tilde{K}_{-}\left(\gamma,-\omega / \beta^{2}\right)\right]_{-} \\
& \quad+\frac{w_{0} \mathrm{e}^{2 \mathrm{i} k_{x} / \beta^{2}}}{(2 \pi)^{2} \mathrm{i}} \frac{\left[\tilde{K}_{-}\left(\gamma,-k_{x} / \beta^{2}\right)\right]_{-}}{K\left(-k_{x} / \beta^{2}\right)}-\sum_{n=0}^{\infty} \frac{\mathcal{A}_{n} \mathrm{e}^{-2 \mathrm{i} \theta_{n}^{-}}}{\mathrm{i}\left(\theta_{n}^{-}+\omega / \beta^{2}\right)}\left[\tilde{K}_{-}\left(\gamma, \theta_{n}^{-}\right)\right]_{-}
\end{aligned}
$$

In a similar way to $\S \mathrm{A} .1$, we now apply the typical Wiener-Hopf argument. We enforce the unsteady Kutta condition (Ayton, Gill \& Peake 2016) which restricts the pressure at the trailing edge to be finite. Consequently, and using the results of appendix B, the left side of (A 26) decays as $|\gamma| \rightarrow \infty$ in $\mathcal{L}^{+}$and the left side of (A 26) tends to an unknown constant as $|\gamma| \rightarrow \infty$ in $\mathcal{L}^{-}$. Applying analytic continuation and Liouville's theorem determines that this constant must be zero. Accordingly, the coefficient of $\gamma^{-1}$ on the left side of (A 26) must vanish so that

$$
P^{*(2)}=\frac{w_{0} \mathrm{e}^{2 \mathrm{i} k_{x} / \beta^{2}}}{(2 \pi)^{2} \mathrm{i} K_{+}\left(-k_{x} / \beta^{2}\right)} \frac{1}{K_{-}\left(-\omega / \beta^{2}\right)}-\sum_{n=0}^{\infty} \frac{\mathcal{A}_{n} \mathrm{e}^{-2 \mathrm{i} \theta_{n}^{-}}}{\mathrm{i}\left(\theta_{n}^{-}+\omega / \beta^{2}\right)} \frac{K_{-}\left(\theta_{n}^{-}\right)}{K_{-}\left(-\omega / \beta^{2}\right)} .
$$

So that, after substituting in the downstream representation (A 23) and the above expression for the pressure constant, the right side of (A 26) yields

$$
\begin{aligned}
D^{(2)}(\gamma)= & \frac{w_{0}\left(\omega-k_{x}\right) \mathrm{e}^{2 \mathrm{i}\left(\gamma+k_{x} / \beta^{2}\right)}}{(2 \pi \beta)^{2} \mathrm{i}\left(\gamma+k_{x} / \beta^{2}\right)\left(\gamma+\omega / \beta^{2}\right) K_{+}\left(-k_{x} / \beta^{2}\right)} \frac{1}{K_{-}(\gamma)} \\
& -\sum_{n=0}^{\infty} \frac{\mathcal{A}_{n} \mathrm{e}^{2 \mathrm{i}\left(\gamma-\theta_{n}^{-}\right)}}{\mathrm{i}\left(\gamma+\omega / \beta^{2}\right)\left(\gamma-\theta_{n}^{-}\right)} \frac{K_{-}\left(\theta_{n}^{-}\right)}{K_{-}(\gamma)} .
\end{aligned}
$$

It should be noted that the only poles of $D^{(2)}$ in $\mathcal{L}^{+}$are at the zeros of $K_{-}$.

\section{A.3. Solution to third and fourth Wiener-Hopf equations $-D^{(3)}, D^{(4)}$}

Since the integral equations for $\Delta_{0}\left[\phi^{(3)}\right]$ and $\Delta_{0}\left[\phi^{(4)}\right]$ are coupled, we must solve for them simultaneously. Taking a Fourier Transform of (A 3) and applying the boundary conditions for (A $6 a$ ) and (A $7 a$ ) for $n=3,4$ gives

$$
\begin{aligned}
F_{-}^{(3)}(\gamma) & =4 \pi\left[D_{-}^{(3)}(\gamma)+D_{+}^{(3)}(\gamma)\right] K(\gamma), \\
F_{+}^{*(4)}(\gamma) & =4 \pi\left[D_{-}^{*(4)}(\gamma)+D_{+}^{*(4)}(\gamma)\right] K(\gamma),
\end{aligned}
$$

where $F_{-}^{(3)}, D_{-}^{(3)}$ and $D_{+}^{(3)}$ are defined in an analogous way to (A 10a,b) and (A 10c), and $F_{+}^{*(4)}, D_{+}^{*(4)}$ and $D_{-}^{*(4)}$ are defined in an analogous way to (A 18a), (A 18b) 
and (A 18c). Using a similar approach to $\S$ A.2, the upstream boundary condition (A 6b) may be expressed as

$$
D_{-}^{(3)}(\gamma)=\frac{1}{2 \pi \mathrm{i}} \int_{-\infty+\mathrm{i} \tau_{0}}^{\infty+\mathrm{i} \tau_{0}} \frac{D^{(2)}\left(\gamma_{1}\right)+D^{(4)}\left(\gamma_{1}\right)}{\gamma_{1}-\gamma} \mathrm{d} \gamma_{1} .
$$

Consequently, we may express $D_{-}^{(3)}$ in terms of its poles $\theta_{n}^{+}$as

$$
D_{-}^{(3)}(\gamma)=-\sum_{n=0}^{\infty} \frac{\mathcal{B}_{n}}{\gamma-\theta_{n}^{+}},
$$

where $\mathcal{B}_{n}$ are the residues of $D_{-}^{(2)}\left(\gamma_{1}\right)+D_{-}^{(4)}\left(\gamma_{1}\right)$ at $\gamma=\theta_{n}^{+}$. The residues of $D_{-}^{(4)}$ are currently unknown, but the residues of $D_{-}^{(2)}$ are given by

$$
\begin{aligned}
D_{r, k}^{(2)}= & \frac{\mathrm{e}^{2 \mathrm{i} \theta_{k}^{+}}}{K_{-}^{\prime}\left(\theta_{k}^{+}\right)}\left\{\frac{-w_{0}\left(\omega-k_{x}\right) \mathrm{e}^{2 \mathrm{i} k_{x} / \beta^{2}}}{(2 \pi \beta)^{2} \mathrm{i}\left(\theta_{k}^{+}+k_{x} / \beta^{2}\right)\left(\theta_{k}^{+}+\omega / \beta^{2}\right) K_{+}\left(-k_{x} / \beta^{2}\right)}\right. \\
& \left.+\sum_{n=0}^{\infty} \frac{\mathrm{i} \mathcal{A}_{n} \mathrm{e}^{\mathrm{i}\left(\theta_{k}^{+}-\theta_{n}^{-}\right)} K_{-}\left(\theta_{n}^{-}\right)}{\left(\theta_{k}^{+}+\omega / \beta^{2}\right)\left(\theta_{k}^{+}-\theta_{n}^{-}\right)}\right\} .
\end{aligned}
$$

We may now substitute (A 30) into (A 28) to obtain the Wiener-Hopf equation

$$
\frac{F_{-}^{(3)}(\gamma)}{4 \pi K_{-}(\gamma)}+\sum_{n=0}^{\infty} \frac{\mathcal{B}_{n}}{\gamma-\theta_{n}^{+}} K_{+}\left(\theta_{n}^{+}\right)=D_{+}^{(3)}(\gamma) K_{+}(\gamma)-\sum_{n=0}^{\infty} \frac{\mathcal{B}_{n}}{\gamma-\theta_{n}^{+}}\left(K_{+}(\gamma)-K_{+}\left(\theta_{n}^{+}\right)\right) .
$$

The edge conditions are identical to those applied in $\S$ A.1, and we employ the typical Wiener-Hopf argument to obtain

$$
D_{+}^{(3)}(\gamma)=\sum_{n=0}^{\infty} \frac{\mathcal{B}_{n}}{\gamma-\theta_{n}^{+}}\left\{1-\frac{K_{+}\left(\theta_{n}^{+}\right)}{K_{+}(\gamma)}\right\}
$$

Combining this solution with (A 30) yields

$$
D^{(3)}(\gamma)=-\sum_{n=0}^{\infty} \frac{\mathcal{B}_{n}}{\gamma-\theta_{n}^{+}} \frac{K_{+}\left(\theta_{n}^{+}\right)}{K_{+}(\gamma)} .
$$

We proceed to the solution for $D^{(4)}$. In a similar way to $\S$ A.2, we may invert the Fourier transform for the downstream boundary condition (A 7b) to write

$$
\begin{aligned}
D_{+}^{*(4)}(\gamma)= & -\frac{P^{*(4)}}{\mathrm{i}\left(\gamma+\omega / \beta^{2}\right)} \\
& -\frac{1}{2 \pi \mathrm{i}} \int_{-\infty-\mathrm{i} \tau_{1}}^{\infty-\mathrm{i} \tau_{1}} \sum_{n=0}^{\infty} \frac{\mathcal{B}_{n}}{\left(\gamma_{1}-\gamma\right)\left(\gamma_{1}-\theta_{n}^{+}\right)}\left\{1-\frac{K_{+}\left(\theta_{n}^{+}\right)}{K_{+}\left(\gamma_{1}\right)}\right\} \mathrm{e}^{-2 \mathrm{i} \gamma_{1}} \mathrm{~d} \gamma_{1} .
\end{aligned}
$$

This integral can be closed in $\mathcal{L}^{-}$to obtain

$$
D_{+}^{*(4)}(\gamma)=-\frac{P^{*(4)}}{\gamma+\omega / \beta^{2}}-\sum_{n=0}^{\infty} \frac{\mathcal{C}_{n} \mathrm{e}^{-2 i \theta_{n}^{-}}}{\mathrm{i}\left(\theta_{n}^{-}+\omega / \beta^{2}\right)\left(\gamma-\theta_{n}^{-}\right)},
$$


where

$$
\mathcal{C}_{n}=\sum_{m=0}^{\infty} \frac{\mathrm{i}\left(\theta_{n}^{-}+\omega / \beta^{2}\right)}{\left(\theta_{m}^{+}-\theta_{n}^{-}\right)} \frac{K_{+}\left(\theta_{m}^{+}\right)}{K_{+}^{\prime}\left(\theta_{n}^{-}\right)} \cdot \mathcal{B}_{m}
$$

After truncation, we may write this system of equations in matrix form

$$
\mathcal{C}=L \mathcal{B}
$$

where

$$
\{\boldsymbol{L}\}_{n, m}=\frac{\mathrm{i}\left(\theta_{n}^{-}+\omega / \beta^{2}\right)}{\left(\theta_{m}^{+}-\theta_{n}^{-}\right)} \frac{K_{+}\left(\theta_{m}^{+}\right)}{K_{+}^{\prime}\left(\theta_{n}^{-}\right)} .
$$

By applying the notation introduced in (A 25), we may express the Wiener-Hopf equation (A 29) in the form

$$
\begin{aligned}
& \frac{F_{+}^{*(4)}(\gamma)}{4 \pi K_{+}(\gamma)}+P^{*(4)}\left[\tilde{K}_{-}\left(\gamma,-\omega / \beta^{2}\right)\right]_{+}+\sum_{n=0}^{\infty} \frac{\left[\tilde{K}_{-}\left(\gamma, \theta_{n}^{-}\right)\right]_{+}}{\mathrm{i}\left(\theta_{n}^{-}+\omega / \beta^{2}\right)} \mathcal{C}_{n} \mathrm{e}^{-2 \mathrm{i} \theta_{n}^{-}} \\
& \quad=D_{-}^{*(4)}(\gamma) K_{-}(\gamma)-P^{*(4)}\left[\tilde{K}_{-}\left(\gamma,-\omega / \beta^{2}\right)\right]_{-}-\sum_{n=0}^{\infty} \frac{\left[\tilde{K}_{-}\left(\gamma, \theta_{n}^{-}\right)\right]_{-}}{\mathrm{i}\left(\theta_{n}^{-}+\omega / \beta^{2}\right)} \mathcal{C}_{n} \mathrm{e}^{-2 \mathrm{i} \theta_{n}^{-}} .
\end{aligned}
$$

Employing the unsteady Kutta condition in (A 34) yields

$$
P^{*(4)}=-\sum_{n=0}^{\infty} \frac{\mathcal{C}_{n} \mathrm{e}^{-2 \mathrm{i} \theta_{n}^{-}}}{\mathrm{i}\left(\theta_{n}^{-}+\omega / \beta^{2}\right)} \frac{K_{-}\left(\theta_{n}^{-}\right)}{K_{-}\left(-\omega / \beta^{2}\right)} .
$$

Finally, applying the downstream boundary condition (A 32) and rearranging (A 34) yields

$$
D^{(4)}(\gamma)=-\sum_{n=0}^{\infty} \frac{\mathcal{C}_{n} \mathrm{e}^{2 \mathrm{i}\left(\gamma-\theta_{n}^{-}\right)}}{\mathrm{i}\left(\theta_{n}^{-}+\omega / \beta^{2}\right)\left(\gamma-\theta_{n}^{-}\right)} \frac{K_{-}\left(\theta_{n}^{-}\right)}{K_{-}(\gamma)} .
$$

We are now able to calculate the residues of $D^{(3)}$ as

$$
\mathcal{B}_{n}=D_{r, n}^{(2)}-\sum_{m=0}^{\infty} \frac{\mathcal{C}_{n} \mathrm{e}^{2 \mathrm{i}\left(\theta_{n}^{+}-\theta_{m}^{-}\right)}}{\mathrm{i}\left(\theta_{n}^{+}+\omega / \beta^{2}\right)\left(\theta_{n}^{+}-\theta_{m}^{-}\right)} \frac{K_{-}\left(\theta_{m}^{-}\right)}{K_{-}^{\prime}\left(\theta_{n}^{+}\right)},
$$

or, in matrix form,

$$
\mathcal{B}=\boldsymbol{D}_{r}+\boldsymbol{F C}
$$

where

$$
\{\boldsymbol{F}\}_{n, m}=\frac{\mathrm{ie}^{2 \mathrm{i}\left(\theta_{n}^{+}-\theta_{m}^{-}\right)}}{\left(\theta_{n}^{+}+\omega / \beta^{2}\right)\left(\theta_{n}^{+}-\theta_{m}^{-}\right)} \frac{K_{-}\left(\theta_{m}^{-}\right)}{K_{-}^{\prime}\left(\theta_{n}^{+}\right)} .
$$

We can combine (A 33) and (A 36) to obtain

$$
(\boldsymbol{I}-\boldsymbol{F L}) \mathcal{B}=D_{r} .
$$


This matrix equation has important physical implications. If the matrix $\boldsymbol{I}-\boldsymbol{F} \boldsymbol{L}$ is not singular then it can be inverted to find $\mathcal{B}$, and then $\mathcal{C}$ by (A 33). On the other hand, if $\boldsymbol{I}-\boldsymbol{F L}$ is singular then there is no solution to the inhomogeneous linear system. However, in such a scenario there is a zero eigenvalue, so the homogeneous linear system $\left(\boldsymbol{D}_{r}=\mathbf{0}\right)$ has a non-trivial solution. Physically speaking, this means that at certain frequencies there exist non-trivial solutions for the scattering by an incident wave of zero amplitude. Such solutions correspond to the so-called 'Parker modes' discovered by Parker (1967), who observed resonance caused by the shedding frequency of the wake coinciding with the natural frequency of the cascade. Koch (1983) extended Parker's results to calculate the resonant frequencies for staggered cascades in a mean flow by using a Wiener-Hopf approach much like the present work. Woodley \& Peake (1999) also deployed the Wiener-Hopf technique to calculate the resonant frequency of tandem cascades. While we do not investigate this further now, the present method could be used to investigate how surface treatments can be used to avoid resonance; the aforementioned references only considered case 0 cascades, whereas our approach can consider more general boundary conditions.

\section{Appendix B. Factorisation of kernel function}

The kernel function is defined as (A 12)

$$
K(\gamma)=\frac{K_{n}(\gamma)}{K_{d}(\gamma)},
$$

where the numerator and denominator functions are respectively

$$
\begin{gathered}
K_{n}(\gamma)=\zeta \sin (s \zeta)-\left(\mu_{0}-\mathrm{i} \mu_{1} \gamma-\mu_{2} \gamma^{2}\right)\left(\cos (s \zeta)-\cos \left(d \gamma+\sigma^{\prime}\right)\right), \\
K_{d}(\gamma)=4 \pi\left(\cos (s \zeta)-\cos \left(d \gamma+\sigma^{\prime}\right)\right) .
\end{gathered}
$$

Our goal is now to obtain a multiplicative factorisation of $K$ into two parts that have no poles or zeros in $\mathcal{L}^{ \pm}$respectively. We restrict our attention to the upper half plane since a corresponding factorisation for the lower half plane can be constructed through symmetry.

\section{B.1. Factorisation of poles of $K$}

Previous work (Peake 1992; Glegg 1999) has factorised the poles of $K$ into the form

$$
K_{d}(\gamma)=K_{d}(0) \prod_{m=-\infty}^{\infty}\left(1-\frac{\gamma}{\lambda_{m}^{+}}\right)\left(1-\frac{\gamma}{\lambda_{m}^{-}}\right),
$$

where

$$
\lambda_{m}^{ \pm}=-f_{m} \sin (\chi) \pm \cos (\chi) \zeta\left(f_{m}\right), \quad f_{m}=\frac{\sigma^{\prime}-2 \pi m}{\Delta} .
$$

The asymptotic behaviour of these poles is

$$
\begin{aligned}
& \lambda_{m}^{+} \sim \lambda_{R}^{(0)+}{ }^{\prime} m+\lambda_{R}^{(2)+}+o(1), \\
& \lambda_{-m}^{+} \sim \lambda_{L}^{(0)+} m+\lambda_{L}^{(2)+}+o(1),
\end{aligned}
$$


as $m \rightarrow \infty$ where

$$
\left.\begin{array}{c}
\lambda_{R}^{(0)+}=\frac{2 \pi \mathrm{ie}^{-\mathrm{i} \chi}}{\Delta}, \quad \lambda_{R}^{(2)+}=\frac{-\mathrm{ie}^{-\mathrm{i} \chi}}{\Delta} \sigma^{\prime}, \\
\lambda_{L}^{(0)+}=\frac{2 \pi \mathrm{ie}^{\mathrm{i} \chi}}{\Delta}, \quad \lambda_{L}^{(2)+}=\frac{\mathrm{ie}^{\mathrm{i} \chi}}{\Delta} \sigma^{\prime} .
\end{array}\right\}
$$

The subscripts $R$ and $L$ indicate that the pole is in the right or left side of $\mathcal{L}^{+}$ respectively.

\section{B.2. Factorisation of zeros of $K$}

We now outline the procedure for factorising the zeros of $K_{n}$. In contrast to previous analyses for case 0 plates (Peake 1992; Glegg 1999), no analytic expression for the zeros is available. In contrast, asymptotic expansions for the large zeros are still tractable, which is useful in two regards. First, the asymptotic expansions provide a good initial guess for a Newton solver to determine some of the actual roots. Second, the asymptotic expansions can be used to bound the growth of the factorised Wiener-Hopf kernel in $\mathcal{L}^{ \pm}$. Understanding this growth is an important step in the Wiener-Hopf procedure, specifically the determination of the relevant entire function.

We focus on the zeros located in the first quadrant of the complex $\gamma$-plane. These roots are labelled as $\theta_{n, R}^{+}$. The asymptotic behaviour of the roots in the other quadrants can be determined by a similar procedure.

Recall the definition of the branch cut of $\zeta$

$$
\zeta=\sqrt{\omega^{2} w^{2}-\gamma^{2}}=\mathrm{e}^{\mathrm{i} \psi_{1} / 2} \mathrm{e}^{\mathrm{i} \psi_{2} / 2}\left|\omega^{2} w^{2}-\gamma^{2}\right|^{1 / 2}
$$

where

$$
\psi_{1}=\arg (\omega w-\gamma), \quad \psi_{2}=\arg (\omega w+\gamma)
$$

and

$$
\pi / 2<\psi_{1}<5 \pi / 2, \quad-\pi / 2<\psi_{2}<3 \pi / 2
$$

Since the $\theta_{n, R}^{+}$are in the first quadrant, we have

$$
\zeta\left(\theta_{n, R}^{+}\right) \sim \mathrm{i} \theta_{n, R}^{+}, \quad \text { as } n \rightarrow \infty .
$$

This leads us to determine the following asymptotic behaviours:

$$
\begin{gathered}
\sin \left(s \zeta\left(\theta_{n, R}^{+}\right)\right) \sim-\frac{1}{2 \mathrm{i}} \mathrm{e}^{s \theta_{n, R}^{+}}, \\
\cos \left(s \zeta\left(\theta_{n, R}^{+}\right)\right) \sim \frac{1}{2} \mathrm{e}^{s \theta_{n, R}^{+}}, \quad \cos \left(d \theta_{n, R}^{+}+\sigma^{\prime}\right) \sim \frac{1}{2} \mathrm{e}^{-\mathrm{i}\left(d \theta_{n, R}^{+}+\sigma^{\prime}\right)} .
\end{gathered}
$$

We now substitute these representations into (B 1) to obtain asymptotic expansions for the roots $\theta_{n, R}^{+}$. Each case must be considered separately, although the asymptotic behaviours are similar. 
Case I boundary condition

For the no-mean-flow boundary condition, the asymptotic behaviour of the roots obeys

$$
\theta_{n, R}^{+} \sim-\mu_{0}\left(1-\exp \left[-\mathrm{i}\left((d-\mathrm{i} s) \theta_{n, R}^{+}+\sigma^{\prime}\right)\right]\right) .
$$

We seek an asymptotic expansion of the first quadrant roots of the classical form

$$
\theta_{n, R}^{+} \sim \sum_{m=0}^{\infty} a_{R}^{(m)} \theta_{R}^{(m)}(n)
$$

where $\theta_{R}^{(m+1)}(n)=o\left(\theta_{R}^{(m)}(n)\right)$ as $n \rightarrow \infty$. In (B 8) we require the linear (left) and exponential (right) terms to match. However, since $\left|\theta_{n, R}^{+}\right| \rightarrow \infty$ as $n \rightarrow \infty$, the exponential term will generally grow at a faster rate than the linear term. Consequently, the real part of the argument of the exponential must be asymptotically small compared to the imaginary part. We therefore expand the roots into real and imaginary parts as $\theta_{n, R}^{+}=\theta_{n, R}^{r+}+\mathrm{i} \theta_{n, R}^{i+}$ and write

$$
d \theta_{n, R}^{i+}-s \theta_{n, R}^{r+}=G(n)
$$

where $G(n)=o\left(\theta_{R}^{(0)}(n)\right)$. Rearranging yields

$$
\theta_{n, R}^{+}=\theta_{n, R}^{+}+\mathrm{i} \theta_{n, R}^{i+}=\left(1+\mathrm{i} \frac{s}{d}\right) \theta_{n, R}^{r+}+\mathrm{i} \frac{G(n)}{d} .
$$

Since the arguments of the left- and right-hand sides of (B 8) must match, we obtain an expression for the imaginary part of the argument of the exponential:

$$
\sigma^{\prime}+d \theta_{n, R}^{r+}+s \theta_{n, R}^{i+}=\chi-2 n \pi+o(1) .
$$

Applying the asymptotic expansion (B 9) and taking the leading-order terms of (B 10) and (B 11) yields

$$
\left.\begin{array}{c}
\theta_{R}^{(0)}(n)=n \\
a_{R}^{(0)}=\frac{2 \pi \mathrm{ie}^{-\mathrm{i} \chi}}{\Delta}
\end{array}\right\}
$$

We may now substitute the expansion for $\theta_{n, R}^{+}$so far into (B 8) to obtain

$$
\frac{2 \pi \mathrm{ie}^{-\mathrm{i} \chi}}{\Delta} n+o(n) \sim-\mu_{0}(1-\exp [G(n)-\mathrm{i} \chi]) .
$$

We now match leading-order terms to obtain

$$
\frac{2 \pi}{\Delta} n=-\mu_{0} \exp [G(n)]
$$

so that

$$
G(n)=\log (n)+\log \left(\frac{-2 \pi}{\mu_{0} \Delta}\right) .
$$


Similarly, taking the leading-order terms in (B 10) and (B 11) yields

$$
\left.\begin{array}{c}
\theta_{R}^{(1)}(n)=\log (n) \\
a_{R}^{(1)}=-\frac{\mathrm{e}^{-\mathrm{i} \chi}}{\Delta},
\end{array}\right\}
$$

and

$$
\begin{gathered}
\theta_{R}^{(2)}(n)=1, \\
\left.a_{R}^{(2)}=\frac{\mathrm{ie}^{-\mathrm{i} \chi}}{\Delta}\left(\frac{\pi}{2}+\chi+\mathrm{i} \log \left(\frac{-2 \pi}{\mu_{0} \Delta}\right)-\sigma^{\prime}\right) \cdot\right\}
\end{gathered}
$$

Substitution of ((B 12), (B 13), (B 14)) into the asymptotic expansion (B 9) yields

$$
\theta_{n, R}^{+} \sim \frac{\mathrm{e}^{-\mathrm{i} \chi}}{\Delta}\left(2 \pi \mathrm{i} n-\log (n)+\mathrm{i}\left(\frac{\pi}{2}+\chi-\sigma^{\prime}\right)-\log \left(\frac{-2 \pi}{\mu_{0} \Delta}\right)+o(1)\right) .
$$

Similar analysis yields the asymptotic behaviour for the roots in the second quadrant as

$$
\theta_{n, L}^{+} \sim \frac{\mathrm{e}^{\mathrm{i} \chi}}{\Delta}\left(2 \pi \mathrm{i} n+\log (n)+\mathrm{i}\left(\frac{\pi}{2}+\chi+\sigma^{\prime}\right)+\log \left(\frac{-2 \pi}{\mu_{0} \Delta}\right)+o(1)\right) .
$$

\section{Case II boundary condition}

In the case of a porous blade in a background flow, the asymptotic behaviour of the roots obeys the equation

$$
1 \sim \mathrm{i} \mu_{1}\left(1-\exp \left[-\mathrm{i}\left((d-\mathrm{i} s) \theta_{n, R}^{+}+\sigma^{\prime}\right)\right]\right) .
$$

We assume an asymptotic expansion of the roots of the form (B 9). Similar reasoning to the previous section yields that the leading-order terms are also given by

$$
a_{R}^{(0)}=\frac{2 \pi \mathrm{ie}^{-\mathrm{i} \chi}}{\Delta}, \quad a_{L}^{(0)}=\frac{2 \pi \mathrm{ie}^{\mathrm{i} \chi}}{\Delta} .
$$

We may now solve (B 17) directly to find the coefficients of the next two orders of the asymptotic expansion as

$$
\begin{aligned}
a_{R}^{(1)}=0, & a_{L}^{(1)}=0, \\
a_{R}^{(2)}=-\frac{\mathrm{e}^{-\mathrm{i} \chi}}{\Delta}\left(\log \left(1+\frac{\mathrm{i}}{\mu_{0}}\right)+\mathrm{i} \sigma^{\prime}\right), & a_{L}^{(2)}=\frac{\mathrm{e}^{\mathrm{i} \chi}}{\Delta}\left(\log \left(1-\frac{\mathrm{i}}{\mu_{0}}\right)+\mathrm{i} \sigma^{\prime}\right) .
\end{aligned}
$$

\section{Case III boundary condition}

For the case III boundary condition, the asymptotic behaviour of the roots obeys

$$
1 \sim \mu_{2} \theta_{n, R}^{+}\left(1-\exp \left[-\mathrm{i}\left((d-\mathrm{i} s) \theta_{n, R}^{+}+\sigma^{\prime}\right)\right]\right) .
$$

Similar analysis to the previous sections possesses an identical asymptotic expansion (up to the terms considered) and we have, at leading order,

$$
a_{R}^{(0)}=\frac{2 \pi \mathrm{ie}^{-\mathrm{i} \chi}}{\Delta}, \quad a_{L}^{(0)}=\frac{2 \pi \mathrm{ie}^{\mathrm{i} \chi}}{\Delta} .
$$

Substitution of (B 20) into (B 19) yields the coefficients of the next two orders of the asymptotic expansion as

$$
\begin{gathered}
a_{R}^{(1)}=0, \quad a_{L}^{(1)}=0, \\
a_{R}^{(2)}=-\frac{\mathrm{i} \sigma^{\prime} \mathrm{e}^{-\mathrm{i} \chi}}{\Delta}, \quad a_{L}^{(2)}=\frac{\mathrm{i} \sigma^{\prime} \mathrm{e}^{\mathrm{i} \chi}}{\Delta} .
\end{gathered}
$$




\section{B.3. Full factorisation of kernel, $K$}

We propose a multiplicative splitting of $K$ of the form:

$$
K(\gamma)=K_{+}(\gamma) K_{-}(\gamma),
$$

where

$$
\begin{gathered}
K_{-}(\gamma)=\mathrm{e}^{E(\gamma)} \frac{\prod_{m=1}^{\infty}\left(1-\gamma / \theta_{m, R}^{+}\right)\left(1-\gamma / \theta_{m, L}^{+}\right)}{\prod_{m=-\infty}^{\infty}\left(1-\gamma / \lambda_{m}^{+}\right)}, \\
K_{+}(\gamma)=\mathrm{e}^{-E(\gamma)} \frac{\prod_{m=1}^{\infty}\left(1-\gamma / \theta_{m, R}^{-}\right)\left(1-\gamma / \theta_{m, L}^{-}\right)}{\prod_{m=-\infty}^{\infty}\left(1-\gamma / \lambda_{m}^{-}\right)} K(0) .
\end{gathered}
$$

The entire function $E$ is included to ensure that $K_{ \pm}$has algebraic growth in $\mathcal{L}^{ \pm}$ respectively. Previous works for case 0 (Peake 1992; Glegg 1999) have derived the form of the entire function $E$ and shown that it is a polynomial. In the remainder of this section, we will show that $E$ vanishes for cases I, II and III.

Asymptotic behaviour of proposed factorisation

We focus on $K_{-}$since the asymptotic behaviour of $K_{+}$follows in an analogous manner. We first note that the infinite product

$$
P_{1}=\prod_{m=1}^{\infty}\left(\frac{a_{R}^{(0)} m+a_{R}^{(2)}}{\theta_{m, R}^{+}}\right)\left(\frac{a_{L}^{(0)} m+a_{L}^{(2)}}{\theta_{m, L}^{+}}\right)
$$

exists. This is seen by employing the asymptotic expansions (B 15) and (B 16) to obtain

$$
\begin{aligned}
& \frac{a_{R}^{(0)} m+a_{R}^{(1)}}{\theta_{m, R}^{+}}=1-\frac{a_{R}^{(1)} \log (m)}{a_{R}^{(0)} m}+o\left(m^{-1}\right), \\
& \frac{a_{L}^{(0)} m+a_{L}^{(1)}}{\theta_{m, L}^{+}}=1-\frac{a_{L}^{(1)} \log (m)}{a_{L}^{(0)} m}+o\left(m^{-1}\right),
\end{aligned}
$$

respectively. Substitution into the product (B 22) yields

$$
P_{1}=\prod_{m=1}^{\infty}\left(1-R \frac{\log (m)}{m}+o\left(m^{-1}\right)\right),
$$

where

$$
R=\frac{a_{R}^{(1)}}{a_{R}^{(0)}}+\frac{a_{L}^{(1)}}{a_{L}^{(0)}}=0,
$$


and the last identity is due to (B 12) and (B 13). Consequently, the products (B 23) and (B 22) exist via the comparison method. Moreover, we note that since

$$
\begin{gathered}
\operatorname{Im}\left[a_{R}^{(0)} m_{1}+a_{R}^{(2)}-\gamma\right]>m_{1} \operatorname{Im}\left[a_{R}^{(0)}\right], \\
\operatorname{Im}\left[a_{L}^{(0)} m_{1}+a_{L}^{(2)}-\gamma\right]>m_{1} \operatorname{Im}\left[a_{L}^{(0)}\right],
\end{gathered}
$$

we can show that the infinite product

$$
P_{2}(\gamma)=\prod_{m=1}^{\infty}\left(\frac{\theta_{m, R}^{+}-\gamma}{a_{R}^{(0)} m+a_{R}^{(2)}-\gamma}\right)\left(\frac{\theta_{m, L}^{+}-\gamma}{a_{L}^{(0)} m+a_{L}^{(2)}-\gamma}\right)
$$

has, at worst, algebraic growth as $\gamma \rightarrow \infty$ in $\mathcal{L}^{-}$, i.e. $\exists \alpha$ s.t. $P(\gamma) \sim \gamma^{\alpha}$, up to a multiplicative constant. We now use $P_{1}$ and $P_{2}$ to calculate the asymptotic behaviour of $K_{-}$by comparison with the product

$$
P(\gamma)=\prod_{m=1}^{\infty} \frac{\left(1-\gamma / \theta_{m, R}^{+}\right) \mathrm{e}^{\gamma / \theta_{m, R}^{+}}\left(1-\gamma / \theta_{m, L}^{+}\right) \mathrm{e}^{\gamma / \theta_{m, L}^{+}}}{\left(1-\gamma /\left(a_{R}^{(0)} m+a_{R}^{(2)}\right)\right) \mathrm{e}^{\gamma / a_{R}^{(0)} m}\left(1-\gamma /\left(a_{L}^{(0)} m+a_{L}^{(2)}\right)\right) \mathrm{e}^{\gamma / a_{L}^{(0)} m}} .
$$

This function may alternatively be written as

$$
P(\gamma)=\exp \left(\gamma \sum_{m=1}^{\infty} \frac{1}{\theta_{m, R}^{+}}-\frac{1}{a_{R}^{(0)} m}+\frac{1}{\theta_{m, L}^{+}}-\frac{1}{a_{L}^{(0)} m}\right) P_{1} P_{2}(\gamma) .
$$

Since $P_{1}$ exists and $P_{2}$ has algebraic growth as $\gamma \rightarrow$ in $\mathcal{L}^{-}$, we have, up to a multiplication constant,

$$
P(\gamma) \sim P_{1} \gamma^{\alpha} \exp \left(\gamma \sum_{m=1}^{\infty} \frac{1}{\theta_{m, R}^{+}}-\frac{1}{a_{R}^{(0)} m}+\frac{1}{\theta_{m, L}^{+}}-\frac{1}{a_{L}^{(0)} m}\right) .
$$

We now calculate the growth of the denominators of $P$. By applying the asymptotic behaviour of the gamma function (Peake 1992, B7 \& B8), we derive the relation

$$
\prod_{m=1}^{\infty}\left(1-\frac{\gamma}{a m+b}\right) \exp \left[\frac{\gamma}{a m}\right] \sim C \exp \left[\frac{\gamma}{a}(\mathcal{E}-1-\log (-a))+\left(\frac{\gamma}{a}-\frac{b}{a}-\frac{1}{2}\right) \log (\gamma)\right],
$$

where $\mathcal{E}$ is the Euler-Mascheroni constant and

$$
C=\frac{-b}{\sqrt{2 \pi}} \Gamma\left(\frac{b}{a}\right)(-a)^{((b / a)-1 / 2)} .
$$

This representation may be substituted into the denominator of (B 26). Rearranging yields the asymptotic behaviour

$$
\begin{aligned}
& \prod_{m=1}^{\infty}\left(1-\frac{\gamma}{\theta_{m, R}^{+}}\right) \mathrm{e}^{\gamma / \theta_{m, R}^{+}}\left(1-\frac{\gamma}{\theta_{m, L}^{+}}\right) \mathrm{e}^{\gamma / \theta_{m, L}^{+}} \\
& \quad \sim B_{2} \gamma^{\alpha} \exp \left[\gamma\left(\sum_{m=1}^{\infty} \frac{1}{\theta_{m, R}^{+}}-\frac{1}{a_{R}^{(0)} m}+\frac{1}{\theta_{m, L}^{+}}-\frac{1}{a_{L}^{(0)} m}\right)\right]
\end{aligned}
$$




$$
\begin{aligned}
& \times \exp \left[\frac{\gamma}{a_{R}^{(0)} a_{L}^{(0)}}\left((\mathcal{E}-1)\left(a_{R}^{(0)}+a_{L}^{(0)}\right)-a_{L}^{(0)} \log \left(-a_{R}^{(0)}\right)-a_{R}^{(0)} \log \left(-a_{L}^{(0)}\right)\right)\right] \\
& \times \exp \left[\left(\gamma\left(\frac{1}{a_{R}^{(0)}}+\frac{1}{a_{L}^{(0)}}\right)-\left(\frac{a_{R}^{(2)}}{a_{R}^{(0)}}+\frac{a_{L}^{(2)}}{a_{L}^{(0)}}\right)-1\right) \log (\gamma)\right],
\end{aligned}
$$

for some constant $B_{2}$. We also note that

$$
\begin{aligned}
& \prod_{m=-\infty}^{\infty}\left(1-\frac{\gamma}{\lambda_{m}^{+}}\right) \mathrm{e}^{\gamma / \lambda_{m}^{+}} \sim B_{3}\left(1-\frac{\gamma}{\lambda_{m}^{+}}\right) \exp \left[\gamma\left(\frac{1}{\lambda_{0}^{+}}+\sum_{\substack{m=-\infty \\
m \neq 0}}^{\infty} \frac{1}{\lambda_{m}^{+}}-\frac{1}{\lambda_{R}^{(0)+}|m|}\right)\right] \\
& \times \exp \left[\frac{\gamma}{\lambda_{R}^{(0)+} \lambda_{L}^{(0)+}}\left((\mathcal{E}-1)\left(\lambda_{R}^{(0)+}+\lambda_{L}^{(0)+}\right)-\lambda_{L}^{(0)+} \log \left(-\lambda_{R}^{(0)+}\right)-\lambda_{R}^{(0)+} \log \left(-\lambda_{L}^{(0)+}\right)\right)\right] \\
& \quad \times \exp \left[\left(\gamma\left(\frac{1}{\lambda_{R}^{(0)+}}+\frac{1}{\lambda_{L}^{(0)+}}\right)-\left(\frac{\lambda_{R}^{(2)+}}{\lambda_{R}^{(0)+}}+\frac{\lambda_{L}^{(2)+}}{\lambda_{L}^{(0)+}}\right)-1\right) \log (\gamma)\right],
\end{aligned}
$$

for some constant $B_{3}$. Noting that $\lambda_{R}^{(0)+}=a_{R}^{(0)}$ and $\lambda_{L}^{(0)+}=a_{L}^{(0)}$, and using (B 7), we obtain

$$
\frac{\prod_{m=1}^{\infty}\left(1-\gamma / \theta_{m, R}^{+}\right)\left(1-\gamma / \theta_{m, L}^{+}\right)}{\prod_{m=-\infty}^{\infty}\left(1-\gamma / \lambda_{m}^{+}\right)} \sim B_{4} \gamma^{c}
$$

for some constant $B_{4}$ and

$$
c=\alpha-\left(\frac{a_{R}^{(2)}}{a_{R}^{(0)}}+\frac{a_{L}^{(2)}}{a_{L}^{(0)}}\right) .
$$

In particular, on a correct selection of the logarithmic branches, we find the following behaviours of the asymptotic factorisations of the kernels (subject to a multiplicative constant):

(i) Case 0,

$$
K_{ \pm}(\gamma) \sim \gamma^{1 / 2}
$$

(ii) Case I,

$$
K_{ \pm}(\gamma) \sim \gamma^{1 / 2}
$$

(iii) Case II,

$$
K_{ \pm}(\gamma) \sim \gamma^{\mp(1 / \pi) \cot ^{-1}\left(\mu_{1}\right)+n}
$$

for some non-negative integer $n$. It is worth noting that the above exponent has appeared before in the porous aerofoil literature. Baddoo, Hajian \& Jaworski (2019) showed that an incompressible, irrotational velocity field near a porous 
edge must scale like $\pm(1 / \pi) \cot ^{-1}(\psi)+n$, where $\psi$ is the porosity parameter. It is not a coincidence that this factor also appears here: through the Wiener-Hopf analysis, the behaviour of the unsteady velocity field at the endpoints of the blade is governed by the behaviour of the factorised kernel at large $\gamma$. Since the edge is self-similar, the singular behaviour is identical for the Laplace and Helmholtz equations.

(iv) Case III:

$$
K_{ \pm}(\gamma) \sim \gamma
$$

\section{Appendix C. Details of Fourier inversion}

The acoustic field is given by

$$
\phi(x, y)=\int_{-\infty}^{\infty} D(\gamma) A(x, y ; \gamma) \mathrm{d} \gamma,
$$

where $A=A_{u}+A_{d}$ and

$$
\begin{gathered}
A_{u}(x, y ; \gamma)=-2 \pi \frac{\mathrm{e}^{\mathrm{i}\left(d \gamma+\sigma^{\prime}\right)} \cos (\zeta y)}{K_{d}(\gamma)} \mathrm{e}^{-\mathrm{i} \gamma x}, \\
A_{d}(x, y ; \gamma)=2 \pi \frac{\cos (\zeta(y-s))}{K_{d}(\gamma)} \mathrm{e}^{-\mathrm{i} \gamma x}
\end{gathered}
$$

and $K_{d}$ is the denominator of $K$ as defined in (B 3). We calculate the above integral by splitting the physical plane into five separate regions, as illustrated in figure 5 . Both $A_{u}$ and $A_{d}$ have poles at the acoustic modes $\gamma=\lambda_{m}^{ \pm}$(B 4) which are the zeros of $K_{d}$. The residues at these points are

$$
\begin{aligned}
& A_{u}^{r}\left(x, y ; \lambda_{m}^{ \pm}\right)=\mp \frac{\zeta_{m}^{ \pm} \mathrm{e}^{ \pm \mathrm{i} s \zeta_{m}^{ \pm}} \cos \left(\zeta_{m}^{ \pm} y\right)}{2 \Delta \sin \left(s \zeta_{m}^{ \pm}\right) \sqrt{\omega^{2} w^{2}-f_{m}^{2}}} \mathrm{e}^{-\mathrm{i} \lambda_{m}^{ \pm} x} \\
& A_{d}^{r}\left(x, y ; \lambda_{m}^{ \pm}\right)= \pm \frac{\zeta_{m}^{ \pm} \cos \left(\zeta_{m}^{ \pm}(y-s)\right)}{2 \Delta \sin \left(s \zeta_{m}^{ \pm}\right) \sqrt{\omega^{2} w^{2}-f_{m}^{2}}} \mathrm{e}^{-\mathrm{i} \lambda_{m}^{ \pm} x},
\end{aligned}
$$

where $f_{m}$ are defined in (B 4) and $\zeta_{m}^{ \pm}=\zeta\left(\lambda_{m}^{ \pm}\right)$. In order to proceed, we split the acoustic potential into four components

$$
\begin{aligned}
\phi(x, y)= & \underbrace{\int_{-\infty}^{\infty} D^{(1,3)}(\gamma) A_{u}(x, y ; \gamma) \mathrm{d} \gamma}_{\phi_{u, u}}+\underbrace{\int_{-\infty}^{\infty} D^{(1,3)}(\gamma) A_{d}(x, y ; \gamma) \mathrm{d} \gamma}_{\phi_{u, d}} \\
& +\underbrace{\int_{-\infty}^{\infty} D^{(2,4)}(\gamma) A_{u}(x, y ; \gamma) \mathrm{d} \gamma}_{\phi_{d, u}}+\underbrace{\int_{-\infty}^{\infty} D^{(2,4)}(\gamma) A_{d}(x, y ; \gamma) \mathrm{d} \gamma}_{\phi_{d, d}},
\end{aligned}
$$

where $D^{(i, j)}=D^{(i)}+D^{(j)}$, and evaluate each contribution individually. The poles of the integrand, and relevant contours of integration, are illustrated in figure 14.

We first calculate

$$
\begin{aligned}
\phi_{u, u}(x, y) & =\int_{-\infty}^{\infty} D^{(1,3)}(\gamma) A_{u}(x, y ; \gamma) \mathrm{d} \gamma \\
& =-2 \pi \int_{-\infty}^{\infty}\left\{D^{(1,3)}(\gamma) K_{+}(\gamma)\right\} K_{-}(\gamma) \frac{\mathrm{e}^{\mathrm{i}(d-x) \gamma+\mathrm{i} \sigma} \cos (\zeta y)}{K_{n}(\gamma)} \mathrm{d} \gamma .
\end{aligned}
$$




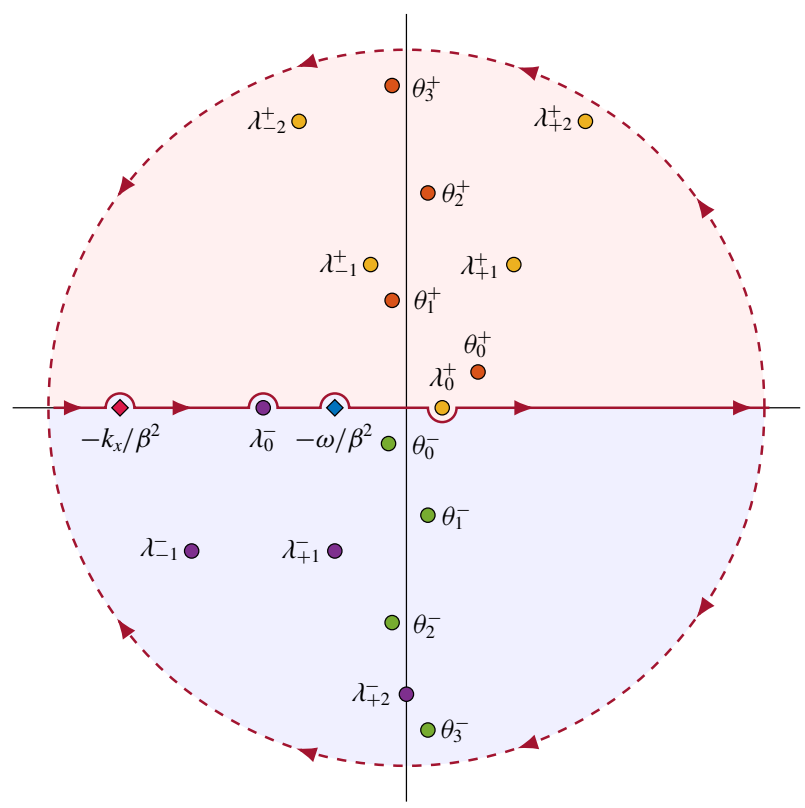

FIGURE 14. Illustration of the locations of poles in the complex $\gamma$-plane, and the relevant contours of integration.

Inspection of (3.11) shows that $\left|D^{(1,3)}(\gamma) K_{+}(\gamma)\right| \rightarrow 0$ as $|\gamma| \rightarrow \infty$ except at the poles at $\theta_{n}^{+}$and $-k_{x} / \beta^{2}$. Consequently, we may close the above integral in $\mathcal{L}^{+}$if $x<d$ and in $\mathcal{L}^{-}$if $x>d$. Accordingly, we obtain for $x<d$

$$
\phi_{u, u}(x, y)=-2 \pi \mathrm{i} \sum_{n=0}^{\infty} \mathcal{B}_{n} A_{u}\left(x, y ; \theta_{n}^{+}\right)+2 \pi \mathrm{i} \sum_{m=-\infty}^{\infty} D^{(1,3)}\left(\lambda_{m}^{+}\right) A_{u}^{r}\left(x, y ; \lambda_{m}^{+}\right),
$$

and for $x>d$,

$$
\phi_{u, u}(x, y)=-2 \pi \sum_{n=0}^{\infty} \frac{\mathcal{A}_{n}+\mathcal{C}_{n}}{\theta_{n}^{-}+\omega / \beta^{2}} A_{u}\left(x, y ; \theta_{n}^{-}\right)-\frac{w_{0} A_{u}\left(x, y ;-k_{x} / \beta^{2}\right)}{2 \pi K\left(-k_{x} / \beta^{2}\right)} .
$$

We proceed by considering

$$
\begin{aligned}
\phi_{u, d}(x, y) & =\int_{-\infty}^{\infty} D^{(1,3)}(\gamma) A_{d}(x, y ; \gamma) \mathrm{d} \gamma \\
& =-2 \pi \int_{-\infty}^{\infty}\left\{D^{(1,3)}(\gamma) K_{+}(\gamma)\right\} K_{-}(\gamma) \frac{-\mathrm{e}^{-\mathrm{i} \gamma x} \cos (\zeta(y-s))}{K_{n}(\gamma)} \mathrm{d} \gamma
\end{aligned}
$$

Using a similar argument to the analysis for (C 1), we close the above integral in $\mathcal{L}^{-}$ if $x>0$ in $\mathcal{L}^{+}$if $x<0$. Consequently, for $x>0$ we obtain

$$
\phi_{u, d}(x, y)=-2 \pi \sum_{n=0}^{\infty} \frac{\mathcal{A}_{n}+\mathcal{C}_{n}}{\theta_{n}^{-}+\omega / \beta^{2}} A_{d}\left(x, y ; \theta_{n}^{-}\right)-\frac{w_{0} A_{d}\left(x, y ;-k_{x} / \beta^{2}\right)}{2 \pi K\left(-k_{x} / \beta^{2}\right)},
$$


and for $x<0$ we obtain

$$
\phi_{u, d}(x, y)=-2 \pi \mathrm{i} \sum_{n=0}^{\infty} \mathcal{B}_{n} A_{d}\left(x, y ; \theta_{n}^{+}\right)+2 \pi \mathrm{i} \sum_{m=-\infty}^{\infty} D^{(1,3)}\left(\lambda_{m}^{+}\right) A_{d}^{r}\left(x, y ; \lambda_{m}^{+}\right) .
$$

We now consider

$$
\begin{aligned}
\phi_{d, u}(x, y) & =\int_{-\infty}^{\infty} D^{(2,4)}(\gamma) A_{u}(x, y ; \gamma) \mathrm{d} \gamma \\
& =-2 \pi \int_{-\infty}^{\infty}\left\{D^{(2,4)}(\gamma) \mathrm{e}^{-2 \mathrm{i} \gamma} K_{-}(\gamma)\right\} K_{+}(\gamma) \frac{\mathrm{e}^{\mathrm{i}(d+2-x) \gamma+\mathrm{i} \sigma^{\prime}} \cos (\zeta y)}{K_{n}(\gamma)} \mathrm{d} \gamma
\end{aligned}
$$

Following a similar argument to $(\mathrm{C} 1),\left|D^{(2,4)}(\gamma) \mathrm{e}^{-2 \mathrm{i} \gamma} K_{-}(\gamma)\right| \rightarrow 0$ as $|\gamma| \rightarrow \infty$ except at the poles at $-\omega / \beta^{2},-k_{x} / \beta^{2}$ and $\theta_{n}^{ \pm}$. Consequently, we close the integral in $\mathcal{L}^{+}$if $x<d+2$ and in $\mathcal{L}^{-}$if $x>d+2$. For $x<d+2$ we obtain

$$
\phi_{d, u}(x, y)=2 \pi \mathrm{i} \sum_{n=0}^{\infty} \mathcal{B}_{n} A_{u}\left(x, y ; \theta_{n}^{+}\right)
$$

and for $x>d+2$ we obtain

$$
\begin{aligned}
\phi_{d, u}(x, y)= & -2 \pi \sum_{n=0}^{\infty} \frac{\mathcal{A}_{n}+\mathcal{C}_{n}}{\theta_{n}^{-}+\omega / \beta^{2}} A_{u}\left(x, y ; \theta_{n}^{-}\right)-2 \pi \mathrm{i} P A_{u}\left(x, y ;-\omega / \beta^{2}\right) \\
& +\frac{w_{0} A_{u}\left(x, y ;-k_{x} / \beta^{2}\right)}{2 \pi K\left(-k_{x} / \beta^{2}\right)}-2 \pi \mathrm{i} \sum_{m=-\infty}^{\infty} D^{(2,4)}\left(\lambda_{m}^{-}\right) A_{u}^{r}\left(x, y ; \lambda_{m}^{-}\right) .
\end{aligned}
$$

The final integral is

$$
\begin{aligned}
\phi_{d, d}(x, y) & =\int_{-\infty}^{\infty} D^{(2,4)}(\gamma) \mathrm{e}^{-\mathrm{i} \gamma x} A_{d}(x, y ; \gamma) \mathrm{d} \gamma \\
& =-2 \pi \int_{-\infty}^{\infty}\left\{D^{(2,4)}(\gamma) \mathrm{e}^{-2 \mathrm{i} \gamma} K_{-}(\gamma)\right\} K_{+}(\gamma) \frac{-\mathrm{e}^{\mathrm{i}(2-x) \gamma} \cos (\zeta(y-s))}{K_{n}(\gamma)} \mathrm{d} \gamma
\end{aligned}
$$

Using similar arguments to the previous integrals, we close the above integral in $\mathcal{L}^{+}$ if $x<2$ and in $\mathcal{L}^{-}$if $x>2$. Consequently, for $x<2$ we obtain

$$
\phi_{d, d}(x, y)=2 \pi \mathrm{i} \sum_{n=0}^{\infty} \mathcal{B}_{n} A_{d}\left(x, y ; \theta_{n}^{+}\right)
$$

and for $x>2$ we obtain

$$
\begin{aligned}
\phi_{d, d}(x, y)= & 2 \pi \sum_{n=0}^{\infty} \frac{\mathcal{A}_{n}+\mathcal{C}_{n}}{\theta_{n}^{-}+\omega / \beta^{2}} A_{d}\left(x, y ; \theta_{n}^{-}\right)+2 \pi \mathrm{i} P A_{d}\left(x, y ;-\omega / \beta^{2}\right) \\
& +\frac{w_{0} A_{d}\left(x, y ;-k_{x} / \beta^{2}\right)}{2 \pi K\left(-k_{x} / \beta^{2}\right)}-2 \pi \mathrm{i} \sum_{m=-\infty}^{\infty} D^{(2,4)}\left(\lambda_{m}^{-}\right) A_{d}^{r}\left(x, y ; \lambda_{m}^{-}\right) .
\end{aligned}
$$

Summing the contributions from each integral yields the full Fourier inversion in $\S 3.1$. 


\section{REFERENCES}

AYton, L. J. 2016 Acoustic scattering by a finite rigid plate with a poroelastic extension. J. Fluid Mech. 791, 414-438.

Ayton, L. J., Gill, J. R. \& Peake, N. 2016 The importance of the unsteady Kutta condition when modelling gust-aerofoil interaction. J. Sound Vib. 378, 28-37.

BADDoo, P. J. \& AYTON, L. J. 2020 An analytic solution for gust-cascade interaction noise including effects of realistic aerofoil geometry. J. Fluid Mech. 886, A1.

BADDOO, P. J., HAJIAN, R. \& JAWORSKI, J. W. 2019 Unsteady aerodynamics of porous aerofoils. arXiv:1911.07382.

Bendali, A., Fares, M'B., Piot, E.\& Tordeux, S. 2013 Mathematical justification of the Rayleigh conductivity model for perforated plates in acoustics. SIAM J. Appl. Maths 73 (1), 438-459.

Bouley, S., François, B., Roger, M., Posson, H. \& Moreau, S. 2017 On a two-dimensional mode-matching technique for sound generation and transmission in axial-flow outlet guide vanes. J. Sound Vib. 403, 190-213.

BRAmbley, E. J. 2009 Fundamental problems with the model of uniform flow over acoustic linings. J. Sound Vib. 322 (4-5), 1026-1037.

BRAndÃo, R. \& SChnitzer, O. 2020 Acoustic impedance of a cylindrical orifice. J. Fluid Mech. 892, A7.

Cavalieri, A. V. G., Wolf, W. R. \& JaWorski, J. W. 2016 Numerical solution of acoustic scattering by finite perforated elastic plates. Proc. R. Soc. Lond. A 472, 20150767.

Crighton, D. G. \& Leppington, F. G. 1970 Scattering of aerodynamic noise by a semi-infinite compliant plate. J. Fluid Mech. 43 (4), 721-736.

Delves, L. M. \& Lyness, J. N. 1967 A numerical method for locating the zeros of an analytic function. Maths Comput. 21 (100), 543-560.

Evers, I. \& PEAKe, N. 2002 On sound generation by the interaction between turbulence and a cascade of airfoils with non-uniform mean flow. J. Fluid Mech. 463, 25-52.

FANG, J. \& ATASSI, H. M. 1993 Compressible flows with vortical disturbances around a cascade of loaded airfoils. In Unsteady Aerodyn. Aeroacoustics, Aeroelasticity Turbomachines Propellers, pp. 149-176. Springer.

Geyer, T., SARRADJ, E. \& FritzSChe, C. 2010 Measurement of the noise generation at the trailing edge of porous airfoils. Exp. Fluids 48 (2), 291-308.

GlegG, S. A. L. 1999 The response of a swept blade row to a three-dimensional gust. J. Sound Vib. 227 (1), 29-64.

GlegG, S. A. L. \& Devenport, W. J. 2017 Aeroacoustics of Low Mach Number Flows: Fundamentals, Analysis, and Measurement. Academic Press.

Graham, R. R. 1934 The silent flight of owls. J. R. Aero. Soc. 38 (286), 837-843.

HALl, K. C. 1997 Exact solution to category 3 problems: turbomachinery noise. In Second Comput. Aeroacoustics $(C A A)$ Work. Benchmark Probl., pp. 41-43. Springer.

Hall, K. C., Kielb, R. E. \& Thomas, J. P. 2006 Unsteady Aerodynamics, Aeroacoustics and Aeroelasticity of Turbomachines. Kluwer Academic Publishers.

Howe, M. S. 1998 Acoustics of Fluid-structure Interactions. Cambridge University Press.

Howe, M. S., ScotT, M. I. \& Sipcic, S. R. 1996 The influence of tangential mean flow on the Rayleigh conductivity of an aperture. Proc. R. Soc. Lond. A 452 (1953), 2303-2317.

JAWORSKI, J. W. \& PEAKE, N. 2013 Aerodynamic noise from a poroelastic edge with implications for the silent flight of owls. J. Fluid Mech. 723, 456-479.

Jaworski, J. W. \& PeAKe, N. 2020 Aeroacoustics of silent owl flight. Annu. Rev. Fluid Mech. 52, $395-420$.

Kisil, A. \& Ayton, L. J. 2018 Aerodynamic noise from rigid trailing edges with finite porous extensions. J. Fluid Mech. 836, 117-144.

Koch, W. 1983 Resonant acoustic frequencies of flat plate cascades. J. Sound Vib. 88 (2), 233-242.

Leppington, F. G. 1977 The effective compliance of perforated screens. Mathematika 24 (02), $199-215$.

Lighthill, M. J. 1958 An Introduction to Fourier Analysis and Generalised Functions. Cambridge University Press. 
Maierhofer, G. \& Peake, N. 2020 Wave scattering by an infinite cascade of non-overlapping blades. J. Sound Vib. 481, 115418.

Myers, M. K. 1980 On the acoustic boundary condition in the presence of flow. J. Sound Vib. 71 (3), 429-434.

Noble, B. 1958 Methods Based on the Wiener-Hopf Technique For the Solution of Partial Differential Equations. Pergamon Press.

PARKER, R. 1967 Resonance effects in wake shedding from compressor blading. J. Sound Vib. 6 (3), 302-309.

Peake, N. 1992 The interaction between a high-frequency gust and a blade row. J. Fluid Mech. 241, 261-289.

PeAKe, N. \& PARRY, A. B. 2012 Modern challenges facing turbomachinery aeroacoustics. Annu. Rev. Fluid Mech. 44 (1), 227-248.

Posson, H., Roger, M. \& Moreau, S. 2010 On a uniformly valid analytical rectilinear cascade response function. J. Fluid Mech. 663, 22-52.

SAIZ, G. 2008 Turbomachinery aeroelasticity using a time-linearised multi blade-row approach. $\mathrm{PhD}$ thesis, Imperial College London.

Strutt, J. W. 1870 On the theory of resonance. Phil. Trans. R. Soc. Lond. 161, 77-118.

Trefethen, L. N. \& Weideman, J. A. C. 2014 The exponentially convergent trapezoidal rule. SIAM Rev. 56 (3), 385-458.

Woodley, B. M. \& Peake, N. 1999 Resonant acoustic frequencies of a tandem cascade. Part 1. Zero relative motion. J. Fluid Mech. 393, 215-240. 